\title{
The role of eddies in driving the tropospheric response to stratospheric heating perturbations
}

Article

Published Version

Simpson, I. R., Blackburn, M. and Haigh, J. D. (2009) The role of eddies in driving the tropospheric response to stratospheric heating perturbations. Journal of the Atmospheric Sciences, 66 (5). pp. 1347-1365. ISSN 1520-0469 doi: https://doi.org/10.1175/2008JAS2758.1 Available at https://centaur.reading.ac.uk/4470/

It is advisable to refer to the publisher's version if you intend to cite from the work. See Guidance on citing.

Published version at: http://dx.doi.org/10.1175/2008JAS2758.1

To link to this article DOI: http://dx.doi.org/10.1175/2008JAS2758.1

Publisher: American Meteorological Society

Publisher statement: (c) Copyright 2009 of the American Meteorological Society. The AMS Copyright Policy is available on the AMS web site at http://www.ametsoc.org

All outputs in CentAUR are protected by Intellectual Property Rights law, including copyright law. Copyright and IPR is retained by the creators or other copyright holders. Terms and conditions for use of this material are defined in the End User Agreement. 


\section{CentAUR}

Central Archive at the University of Reading

Reading's research outputs online 


\title{
The Role of Eddies in Driving the Tropospheric Response to Stratospheric Heating Perturbations
}

\author{
ISLA R. SIMPSON \\ Department of Physics, Imperial College London, London, United Kingdom \\ MiCHAEL BLACKBURN \\ National Centre for Atmospheric Science, University of Reading, Reading, United Kingdom \\ JOANNA D. HAIGH \\ Department of Physics, Imperial College London, London, United Kingdom
}

(Manuscript received 6 February 2008, in final form 8 October 2008)

\begin{abstract}
A simplified general circulation model has been used to investigate the chain of causality whereby changes in tropospheric circulation and temperature are produced in response to stratospheric heating perturbations. Spinup ensemble experiments have been performed to examine the evolution of the tropospheric circulation in response to such perturbations.

The primary aim of these experiments is to investigate the possible mechanisms whereby a tropospheric response to changing solar activity over the 11-yr solar cycle could be produced in response to heating of the equatorial lower stratosphere. This study therefore focuses on a stratospheric heating perturbation in which the heating is largest in the tropics. For comparison, experiments are also performed in which the stratosphere is heated uniformly at all latitudes and in which it is heated preferentially in the polar region. Thus, the mechanisms discussed have a wider relevance for the impact of stratospheric perturbations on the troposphere.

The results demonstrate the importance of changing eddy momentum fluxes in driving the tropospheric response. This is confirmed by the lack of a similar response in a zonally symmetric model with fixed eddy forcing. Furthermore, it is apparent that feedback between the tropospheric eddy fluxes and tropospheric circulation changes is required to produce the full model response. The quasigeostrophic index of refraction is used to diagnose the cause of the changes in eddy behavior. It is demonstrated that the latitudinal extent of stratospheric heating is important in determining the direction of displacement of the tropospheric jet and storm track.
\end{abstract}

\section{Introduction}

In recent years evidence has accumulated that changes in the stratosphere can have a significant impact on the troposphere and that there are many climate forcings that can potentially have an impact on tropospheric circulation through a stratospheric pathway (Shepherd 2002; Haynes 2005).

Corresponding author address: Isla Simpson, Space and Atmospheric Physics Group, The Blackett Laboratory, Imperial College London, Prince Consort Road, London SW7 2AZ, United Kingdom.

E-mail: isla.simpson05@imperial.ac.uk
One such forcing is the irradiance change associated with the 11-yr solar cycle. Several studies have shown consistent changes in tropospheric temperature and circulation over the cycle involving a temperature response that is nonuniform in latitude, together with altered mean meridional circulation and zonal wind anomalies (Haigh 2003; Haigh et al. 2005, hereafter HBD05; Crooks and Gray 2005; Salby and Callaghan 2006; Gleisner and Thejll 2003). HBD05 showed that at solar maximum there is a weakening and poleward shift of the midlatitude jets compared to solar minimum, which consists of a reduction in zonal-mean zonal wind of over $1 \mathrm{~m} \mathrm{~s}^{-1}$ on the equatorward side of the jet and an acceleration of a few tenths of $1 \mathrm{~m} \mathrm{~s}^{-1}$ on the poleward side of the jet. A 
similar pattern is found in atmospheric GCM studies of the response to enhanced solar UV (Haigh 1996, 1999; Larkin et al. 2000; Matthes et al. 2006), and these also suggest a change in the mean meridional circulation, consisting of a weakening of the dominant winter Hadley cell and a poleward shift of the summer Hadley and Ferrell cells. These circulation changes are accompanied by a tropospheric temperature response that consists of a banded increase of over $0.5 \mathrm{~K}$ in midlatitudes (between $\sim 20^{\circ}$ and $60^{\circ}$ latitude) in thermal wind balance with the zonal wind accelerations (Haigh 2003; Crooks and Gray 2005; Lu et al. 2007).

One possible mechanism for the production of this tropospheric solar signal is through a dynamical response to enhanced heating that occurs in the lower stratosphere (Haigh 1996; Kristjánsson et al. 2002; Baldwin and Dunkerton 2005). Although the direct radiative effect of the solar cycle is larger in the upper stratosphere, there is a secondary maximum in the temperature response found in the lower stratosphere, with an increase in temperature on the order of $1 \mathrm{~K}$ found over the equatorial latitudes (Labitzke et al. 2002; Crooks and Gray 2005; Haigh 2003).

To investigate whether lower stratospheric heating over the solar cycle could produce the observed tropospheric response, HBD05 looked at the effect of applying heating perturbations to the stratosphere of a simplified GCM (sGCM). It was found that a tropospheric signal similar in structure to that seen over the solar cycle could be produced in response to heating of the stratosphere, which is largest at the equator. Because this is similar in structure to the observed lower stratospheric temperature response to the 11 -yr cycle, it lends support to the idea that the tropospheric circulation changes could be produced through a response to the heating of the lower stratosphere.

There are many other climate forcings that could also have an impact on tropospheric circulation through a change in temperature of the lower stratosphere, and several authors have investigated the effect of applying different temperature perturbations to the stratospheres of sGCMs (HBD05; Polvani and Kushner 2002; Kushner and Polvani 2004, 2006; Williams 2006; Lorenz and DeWeaver 2007). In each of these studies, a stratospheric temperature perturbation produces a shift in position and strength of the tropospheric jets, the sign of which appears to depend on the sign and latitudinal distribution of the applied temperature perturbation. For example, HBD05 and Williams (2006) have shown that in response to a latitudinally uniform increase in temperature of the stratosphere (and corresponding lowering of the tropopause), there is an equatorward shift of the midlatitude jets. Analogously, Lorenz and DeWeaver
(2007) found a strengthening and poleward shift of the midlatitude jets in response to a cooling of the stratosphere, such as predicted in response to increased greenhouse gas concentrations.

The work of Polvani and Kushner (Polvani and Kushner 2002; Kushner and Polvani 2004, 2006) has focused on investigating the mechanisms involved in the tropospheric response to cooling of the polar stratosphere of an sGCM. They found a poleward shift of the tropospheric jet in response to the stratospheric cooling and demonstrated the importance of changing eddy momentum fluxes in producing the tropospheric response. They also found that the full tropospheric response could not be produced without internal tropospheric eddy feedbacks. HBD05 also demonstrated the importance of altered eddy momentum fluxes in maintaining their tropospheric response to stratospheric heating perturbations. However, it is not yet known exactly how changes in lower stratospheric temperature influence the eddies to produce such a response.

Moreover, it is not clear what determines the direction of the tropospheric jet shift. Lorenz and DeWeaver (2007) investigated whether the tropospheric response was more sensitive to changes in the height of the tropopause or changes in the meridional temperature gradient. They applied a heating perturbation of $20^{\circ}$ latitude width and 150-hPa height at various positions in the latitude-pressure plane and found that the sign of the tropospheric jet shift changes dramatically as the heating perturbation is moved from below to above the tropopause, suggesting that changes in tropopause height are important. However, they also find a change in the sign of the tropospheric response as the heating moves meridionally either above or below the tropopause.

The above experiments have demonstrated that in response to stratospheric heating perturbations there is a shift in tropospheric circulation associated with changes in eddy momentum fluxes. The response appears to depend on the sign of the heating perturbation and also on its meridional distribution. But it is not yet clear exactly how such a response is produced. In this paper we address some of these issues as we investigate in more detail the mechanisms involved in producing the tropospheric response to perturbations in lower stratospheric temperature. We carry out spinup ensemble experiments using the same sGCM as HBD05 to investigate the evolution of the response. Our analysis is first presented in the context of the equatorial heating case because this most closely resembles the solar cycle response. We then compare with the uniform and polar heating cases and with experiments using a zonally symmetric model to demonstrate the importance of changing eddy fluxes. Section 2 describes the model and 
experiments and section 3 presents the results of the 3D model runs with equatorial heating. These are compared with the results of the zonally symmetric model in section 4 . A brief comparison is made with the uniform and polar heating cases in section 5, and discussion and conclusions follow in section 6 .

\section{Model and experimental design}

\section{a. The model}

The sGCM used in the following experiments is the same as that used in HBD05. It has the spectral dynamical core described by Hoskins and Simmons (1975), with modification to include the angular momentumconserving vertical discretization of Simmons and Burridge (1981) while retaining the original sigma coordinate. Triangular truncation at wavenumber 42 is used. There are 15 levels between the surface and $\sigma=$ 0.0185 with the level spacing chosen to give good resolution in the region of the tropopause, which is crucial for investigations of the tropospheric response to stratospheric heating perturbations. The model sigma levels are as follows: $0.0185,0.0596,0.106,0.152,0.197,0.241,0.287$, $0.338,0.400,0.477,0.569,0.674,0.784,0.887$, and 0.967 .

Although the model includes an accurate representation of large-scale dynamical processes, it has a highly parameterized representation of physical processes. Instead of the moist and radiative parameterizations of a full GCM, the climate is maintained by Newtonian relaxation of the temperature field toward a zonally symmetric equilibrium state (Held and Suarez 1994) on a time scale of 40 days for $\sigma<0.7$ (representing radiation and deep, moist processes) and reducing to 4 days at the equatorial surface (representing the planetary boundary layer). Boundary layer friction is represented by Rayleigh damping of winds below $\sigma=0.7$ with a time scale of 1 day at the surface.

Orography is omitted, so there is no large-scale zonally asymmetric forcing, resulting in relatively weak planetary waves generated only by the internal dynamics. Baroclinic eddies dominate the wave spectrum with peak amplitude at zonal wavenumbers 5 to 7 . These are initiated through a white noise perturbation applied to the surface pressure field at the beginning of each equilibrium integration.

\section{b. Spinup ensemble experiments}

The results of HBD05 have demonstrated the equilibrium response to the application of several different heating perturbations to the stratosphere of the sGCM. In this investigation we carry out spinup ensemble experiments to further investigate the mechanisms involved in transmitting the response of the applied stratospheric heating perturbation to the troposphere below. The stratospheric heating perturbations are applied by altering the Newtonian equilibrium temperature field (see HBD05 for details). The E5 case consists of an increase in stratospheric temperature of $5 \mathrm{~K}$ at the equator decreasing to $0 \mathrm{~K}$ at the poles, with the tropospheric relaxation temperature unchanged. The U5 case consists of a uniform increase of $5 \mathrm{~K}$ throughout the stratosphere, whereas the P10 case has an increase of $10 \mathrm{~K}$ at the pole decreasing to $0 \mathrm{~K}$ at the equator. Equatorial heating more resembles that seen over the solar cycle, although the applied temperature perturbation is considerably larger than that observed (Haigh 2003; Labitzke et al. 2002). However, it was found by HBD05 that the results were qualitatively independent of the magnitude of the applied heating, with the magnitude of the response varying linearly in the stratosphere and with a slightly larger response than linear in the troposphere. This suggests that although the applied temperature perturbation in the E5 case is large, the mechanisms involved in the tropospheric response will be the same as with a smaller stratospheric heating perturbation. The model experiments are not intended to be a direct simulation of the climate response over the solar cycle, but they can be of use in investigating the mechanisms involved in a tropospheric response to enhanced heating in the stratosphere over the solar cycle. Although we are primarily investigating mechanisms by which the tropospheric response to solar activity is produced, our results apply equally to other situations in which there is a thermal perturbation to the stratosphere, such as the cooling expected with increased greenhouse gas concentrations (Lorenz and DeWeaver 2007). The U5 heating case could be thought of as the opposite of a greenhouse gas stratospheric cooling scenario, whereas the P10 case could be of use in interpreting any circulation changes associated with a warming of the polar stratosphere, such as might occur with ozone recovery (Son et al. 2008).

Each spinup ensemble consists of 200 50-day runs. Each ensemble member starts from different initial conditions taken at 50-day intervals from a control simulation in which no stratospheric heating perturbation was applied. In this way, the starting conditions are different for each ensemble member but remain within the natural variability of the control run. The stratospheric heating perturbation is then switched on and the model is allowed to respond over the following 50-day period. By averaging over the ensemble, a statistical signal emerges from internally generated variability and the evolution in response to the applied heating perturbation is clearly demonstrated. The number of data points has been doubled by averaging over both hemispheres. As was noted in HBD05, this is possible 
because the temporal correlation between the equivalent points in the two hemispheres is very low.

In addition to the spinup ensembles, an equilibrium run has been performed for each heating case. For each of these, the model is spun up from rest with the stratospheric heating perturbation continuously applied and the results taken from a 5000-day average after an initial spinup period of 200 days. ${ }^{1}$

We focus in sections 3 and 4 on the E5 case and then confirm the ideas presented in sections 3 and 4 by comparison with the U5 and P10 experiments in section 5.

\section{Results for the $\mathbf{E} 5$ case}

\section{a. Spinup ensemble evolution}

The spinup ensemble average evolution of zonal mean temperature $\bar{T}$, zonal-mean zonal wind $\bar{u}$, and streamfunction of the zonal mean meridional circulation $\bar{\Psi}$ for the E5 case are shown in Fig. 1. Results are presented as 10-day averages with the difference taken relative to the average of the equivalent 10 days of the control run following the start day of the spinup to limit any apparent evolution that is due to internal variability. The top panels of Fig. 1 show latitude-pressure cross sections of each zonal-mean quantity for the 10000 -day control run, and the bottom panel shows the difference between the 5000-day equilibrium E5 run and the control run.

In the initial 10 days after the perturbation is switched on, there is an increase in the temperature of the stratosphere, with warming that is largest at the equator and decreasing toward the poles. This reduces the reversed latitudinal temperature gradient of the control run and induces poleward flow, which results in a broad increase in vertical wind shear in the stratosphere to maintain thermal wind balance. The tropopause in the equilibrium temperature distribution slopes down from equator to pole (HBD05; Fig. 2a), so the perturbation also increases the meridional temperature gradient equatorward of $\sim 30^{\circ}$ latitude around the $200-\mathrm{hPa}$ level. This induces equatorward flow and an easterly anomaly with decreased vertical wind shear immediately below it at the subtropical tropopause.

As the spinup progresses, a response begins to be seen in the troposphere. This consists of a band of increased

\footnotetext{
${ }^{1}$ The equilibrium response has been calculated from a 5000-day average rather than a 1000-day average as in HBD05. In extending the runs, it was found that a 1000-day average may not be representative of the magnitude of the model response due to variability. The equilibrium response for the U5 case is found to be roughly between $1 / 2$ and $1 / 3$ of the magnitude of the response in HBD05. The magnitude of the E5 response remains similar to that in HBD05.
}

temperature in the midlatitude troposphere centered on $\sim 45^{\circ}$ latitude and a decrease on either side (see Fig. 1). This banded structure is already very similar to the equilibrium response by days 20 to 29 . It continues to intensify through the spinup period, but the equilibrium response has not yet been reached after 50 days.

In thermal wind balance with these tropospheric temperature changes, there is an increased westerly wind on the poleward side of the jet and a decrease on the equatorward side. This corresponds to a weakening and poleward shift of the midlatitude jet. Again, this zonal wind response is small initially but continues to amplify throughout the spinup period while the features in the subtropical upper troposphere migrate slowly poleward leading to a poleward tilt with height.

A response in mean meridional circulation is also apparent near the beginning of the spinup (Fig. 1c). In days 0 to 9 it consists of a weakening of the Hadley cell and the equatorward side of the Ferrell cell, starting in the upper troposphere. This is qualitatively consistent with the response to the temperature gradients directly generated by the stratospheric heating, as already discussed. By days 20 to 29, the meridional circulation has extended throughout the depth of the troposphere and an anomalous indirect circulation has also appeared at high latitudes. This three-cell pattern continues to increase in magnitude as time progresses. The regions of anomalous descent and adiabatic warming coincide with the regions of increased temperature in the troposphere and vice versa. The change in the convergence of poleward eddy heat flux (not shown) acts to oppose these tropospheric temperature changes such that, by equilibrium, there is a balance between the adiabatic heating (cooling) and divergence (convergence) of the poleward eddy heat flux (see HBD05 for the equilibrium poleward eddy heat flux).

It is apparent that there is a balanced response in the troposphere to the stratospheric heating, but how is such a response produced when the temperature perturbation is only applied in the stratosphere? HBD05 demonstrated that changes in the horizontal eddy momentum flux $\left(\overline{u^{\prime} v^{\prime}}\right)$ were crucial in maintaining the anomalous zonal flow against low-level drag. In the following section, we demonstrate not only that the changing eddy momentum fluxes are important in maintaining the anomalous circulations but also that they are instrumental in creating them.

\section{b. The importance of changing eddy momentum fluxes}

The results of the previous section have demonstrated that altered temperature gradients in the stratosphere and around the tropopause region result in zonal wind 

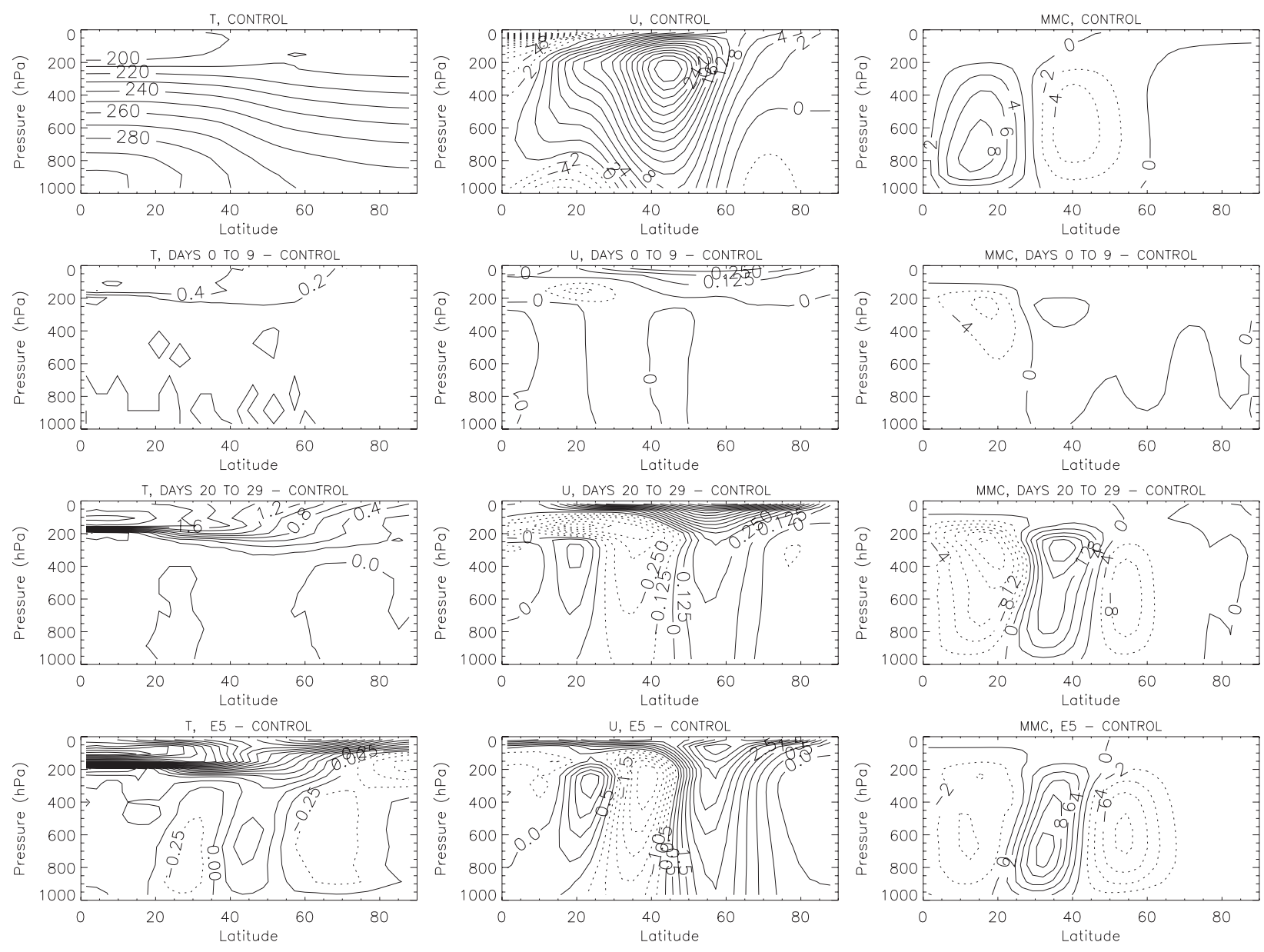

a: ZONAL MEAN T

b: ZONAL MEAN U

c: MMC

FIG. 1. Evolution of zonal mean fields for the E5 case. (top) Control run values; (bottom) difference between the 5000-day average equilibrium values and the control run; (middle) difference between 10-day averages of the spinup and the equivalent 10 days of the control run for days 0 to 9 and 20 to 29. (a) Temperature (K), (b) zonal wind ( $\mathrm{m} \mathrm{s}^{-1}$ ), (c) streamfunction of the mean meridional circulation [(top) $10^{10} \mathrm{~kg} \mathrm{~s}^{-1}$; (middle) $10^{8} \mathrm{~kg} \mathrm{~s}^{-1}$; (bottom) $10^{9} \mathrm{~kg} \mathrm{~s}^{-1}$ ]. Dashed contours are negative. Note the difference in the contour interval among the panels.

accelerations there. Figure 2 demonstrates that this is also accompanied by altered horizontal eddy momentum fluxes, and it becomes apparent when looking at momentum balance that these are important in driving the meridional wind changes in the upper troposphere, which in turn are important in producing the zonal wind anomalies in the lower troposphere. Throughout the following we use "horizontal eddy momentum flux" to refer to momentum flux in the equator to pole direction.

The momentum balance in the conventional Eulerian mean can be written

$$
\begin{aligned}
\frac{\partial \bar{u}}{\partial t}= & f \bar{v}-\frac{1}{a \cos ^{2} \phi} \frac{\partial \overline{u^{\prime} v^{\prime}} \cos ^{2} \phi}{\partial \phi} \\
& -k \bar{u}+\text { ageostrophic terms, }
\end{aligned}
$$

where overlined quantities represent zonal means and dashed quantities represent the deviations from the zonal mean; $a$ is the radius of the earth and $k$ is the boundary layer frictional damping coefficient.

The $\bar{u}$ anomalies relative to $t=0$ can then be given by

$$
\begin{aligned}
\bar{u}(t)-\bar{u}(0)= & \frac{1}{e^{k t}}\left[\int_{0}^{t} e^{k t} f \bar{v} d t\right. \\
& +\int_{0}^{t}-e^{k t} \frac{1}{a \cos ^{2} \phi} \frac{\partial \overline{u^{\prime} v^{\prime}} \cos ^{2} \phi}{\partial \phi} d t \\
& \left.+\int_{0}^{t} e^{k t}(\text { ageostrophic terms }) d t\right] .
\end{aligned}
$$

To derive this solution, the dependence of the ageostrophic terms on $\bar{u}$ has been ignored. Given the good agreement between the sum of the terms on the rhs of Eq. (2) and the $\bar{u}$ anomaly (as will be shown below), this appears to be a reasonable approximation. Note that for $p<700 \mathrm{hPa}, k=0$ and the solution is exact. 

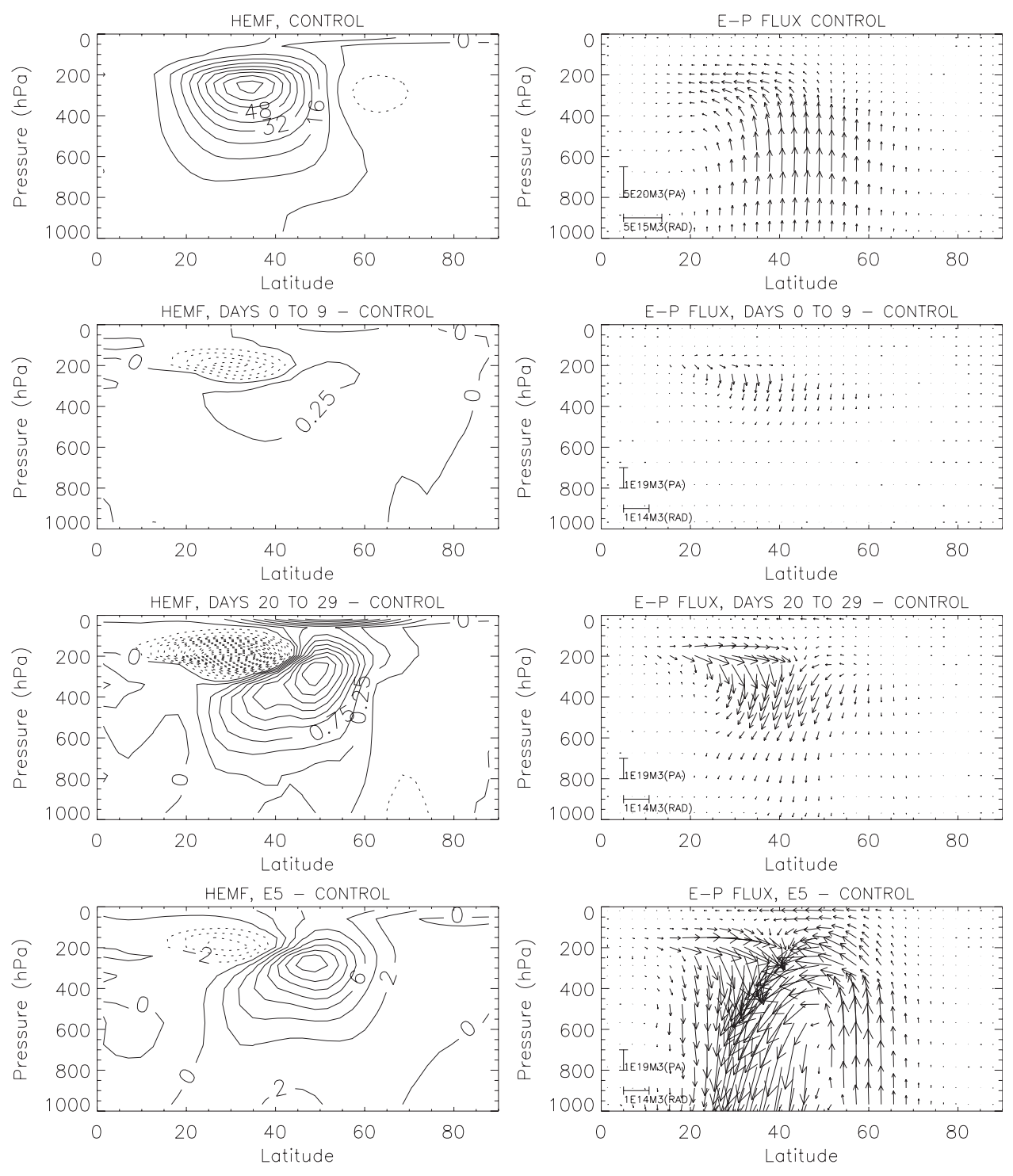

FIG. 2. As in Fig. 1, but for (a) $\overline{u^{\prime} v^{\prime}}$ and (b) E-P flux scaled as in Edmon et al. (1980) for the E5 case. Note the different scale of the E-P flux vectors and the different contour intervals between plots.

Thus there are three terms that act to change $\bar{u}$ : the Coriolis force acting on the anomalous mean meridional wind, the change in horizontal eddy momentum flux convergence, and the ageostrophic terms. Mean meridional wind anomalies arise in response to thermal wind imbalances created directly by the anomalous heating and by anomalous eddy fluxes.

Comparing Figs. 1c and 2a, it is evident that the anomalous mean meridional circulation is in the correct sense to (at least partially) balance the anomalous horizontal eddy momentum flux.

In response to the stratospheric heating, there is a horizontal dipole in the change in horizontal eddy mo- mentum flux, which gives a tripole of forcing in Eq. (1). This tripole corresponds to the latitudes of the three-cell pattern in the meridional circulation anomalies. By days 20 to 29 the increase in horizontal eddy momentum flux on the poleward side of the jet has become more prominent and stretches downward and equatorward into the troposphere.

The altered temperature gradients around the tropopause will tend to drive an anomalous indirect circulation equatorward of $\sim 30^{\circ}$ latitude and a direct circulation poleward of this. These circulations will drive easterly winds equatorward of $30^{\circ}$ latitude and westerly winds poleward of this near $200 \mathrm{hPa}$ via Coriolis torque. 
During days 0 to 9 , the $\overline{u^{\prime} v^{\prime}}$ anomalies result in a region of increased convergence equatorward of around $30^{\circ}$ latitude. This will tend to drive changes in the zonal wind but will also induce a mean meridional circulation opposing these changes. The dipole in momentum flux convergence associated with the negative $\overline{u^{\prime} v^{\prime}}$ anomaly is located so as to enhance the mean meridional circulations and weaken the zonal winds driven directly by the heating. At the highest latitudes there is an increased convergence of $\overline{u^{\prime} v^{\prime}}$, but this does not initially dominate over the effect of the altered temperature gradients. By days 20 to 29 the positive part in the dipole of eddy momentum flux has become much more prominent, and in the region poleward of $\sim 50^{\circ}$ latitude this results in an anomalous indirect circulation.

Thus, regions of increased (decreased) convergence of horizontal eddy momentum flux coincide with regions of decreased (increased) meridional wind, as would be expected from Eq. (1). This is further demonstrated in the top panels of Fig. 3, which show time series of the change in each of the forcing terms in Eq. (2) over the spinup, along with their sum and the zonal wind anomaly (difference between spinup and control run at time $t$ ), averaged between $700 \mathrm{hPa}$ and the top of the model. Figure $3 \mathrm{a}$ shows the average over $34^{\circ}-37^{\circ}$ latitude (the region of decreased $\bar{u}$ on the equatorward side of the jet) and Fig. $3 \mathrm{~b}$ shows the average over $54^{\circ}-$ $57^{\circ}$ latitude (in the region of acceleration on the poleward side of the jet). This demonstrates that over these latitudes, in the vertical average outside the boundary layer, the dominant balance is between anomalous horizontal eddy momentum flux and anomalous $f \bar{v}$, with the imbalance giving a net acceleration in $\bar{u}$.

In almost all regions the change in horizontal eddy momentum flux is considerably larger than the change in vertical eddy momentum flux. However, the latter does become important around the $30^{\circ}$ latitude region, where the change in horizontal eddy momentum flux convergence approaches zero. Thus, the vertical eddy momentum flux is important in determining the exact latitude of zero meridional-zonal wind changes in the subtropics. At certain pressure levels on the equatorward side of the jet the ageostrophic terms become important, but their contribution to the change in $\bar{u}$ cancels out when integrating over the upper half of the atmosphere, leaving the dominant balance between the horizontal eddy momentum flux and the $f \bar{v}$ anomalies, as shown.

The middle panel of Fig. 3 shows the anomalous $f \bar{v}$ vertically integrated over the free atmosphere and within the frictional boundary layer (i.e., above and below $700 \mathrm{hPa}$ ), averaged over the same latitudes [note this is the instantaneous $f \bar{v}$ acceleration in Eq. (1), not the time-integrated value from Eq. (2) shown in the upper and lower panels]. The anomalous meridional wind at upper levels clearly mirrors that at lower levels, so that meridional wind anomalies produced in response to eddy momentum flux changes in the upper troposphere are balanced by meridional wind anomalies in the lower troposphere of opposite sign, as expected through downward control (Haynes et al. 1991).

Comparison of Figs. $1 \mathrm{~b}$ and $1 \mathrm{c}$ shows that the regions of anomalous poleward meridional wind in the lower troposphere correspond to regions of increased westerly zonal wind and vice versa, suggesting that the $f \bar{v}$ term at lower levels gives rise to the zonal wind accelerations there.

This is confirmed in the lower panel of Fig. 3, which shows each of the terms in Eq. (2) for the region of acceleration on the poleward side of the jet and the region of deceleration on the equatorward side of the jet, but this time averaged from $700 \mathrm{hPa}$ to the surface. Here it is $f \bar{v}$ that is the dominant contribution to the change in $\bar{u}$. Thus, changes in horizontal eddy momentum flux in the upper troposphere drive altered meridional circulations that lead to zonal wind accelerations in the lower troposphere. Figure 3 suggests that this occurs in two stages with an initial slow acceleration of the zonal wind in the first 20 days followed by faster changes, particularly on the poleward side of the jet.

The vertically integrated momentum budget has also been analyzed. In this diagnostic, the terms involving the Coriolis force acting on the mean meridional wind cancel. Additionally, the terms involving surface pressure variations and the surface momentum flux associated with the resolved flow are small and can be neglected. The vertically integrated momentum budget then becomes

$$
\frac{1}{g} \frac{\partial}{\partial t} \int_{0}^{P_{s}} \bar{u} d p=C_{\mathrm{ZONAL}}+C_{\mathrm{EDDY}}-\bar{\tau}_{S \lambda},
$$

where $C_{\text {ZONAL }}$ is the zonally averaged convergence of the poleward flux of westerly momentum by the mean circulation $(\bar{u} \bar{v}), C_{\text {EDDY }}$ is the zonally averaged convergence of the poleward flux of westerly momentum due to the eddies $\left(\overline{u^{\prime} v^{\prime}}\right)$, and $\bar{\tau}_{S \lambda}$ is the zonally averaged surface stress (See HBD05 for details).

The vertically integrated momentum budget for the E5 spinup and equilibrium is shown in Fig. 4. The 10 000-day average of the control run is shown, along with the anomalies for days 0 to 9 and 20 to 29 of the spinup and the anomalies for the E5 equilibrium run.

This demonstrates that in the region of zonal wind acceleration on the poleward side of the jet (between $\sim 45$ and $70^{\circ}$ ), there is a significant increase in horizontal 

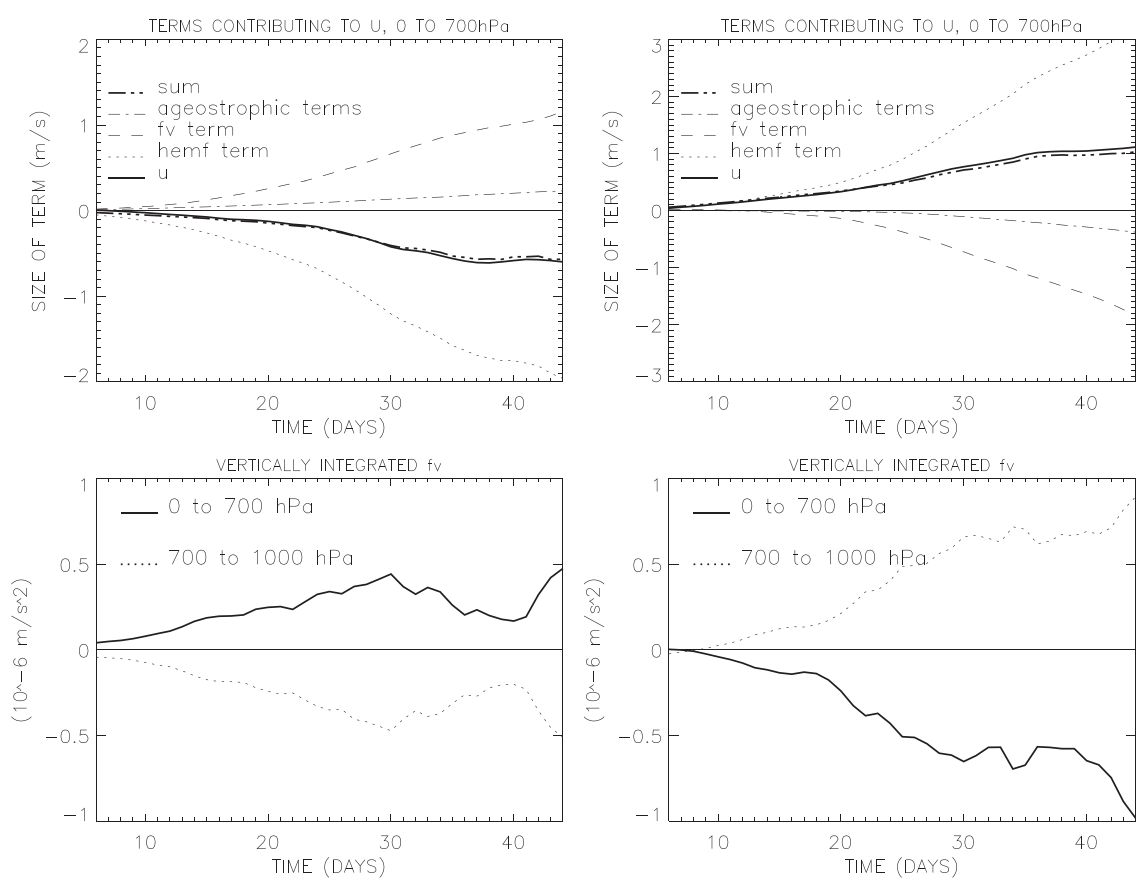

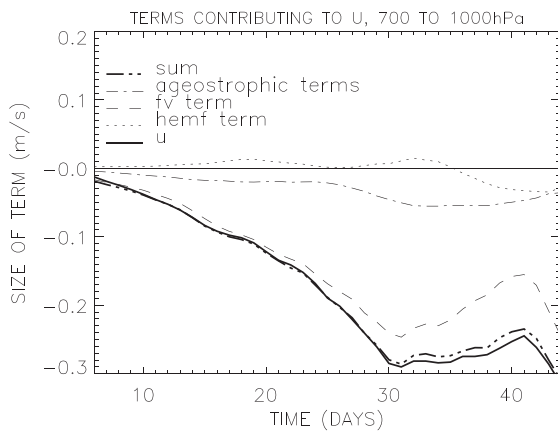

(a) averaged from 34 to $37 \mathrm{deg}$ lat

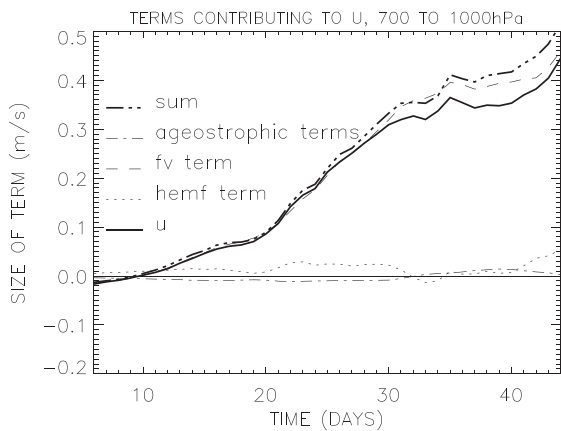

(b) averaged from 54 to $57 \mathrm{deg}$ lat

FIG. 3. (top) Eleven-day running means of the change in each of the terms in Eq. (2) along with their sum and the difference in $\bar{u}$ from the control run [hemf $=2$ nd term in Eq. (2)], averaged from the top of the model to $700 \mathrm{hPa}$; (middle) 11-day running means of $f \bar{v}$ anomaly vertically integrated from 0 to $700 \mathrm{hPa}$ and from $700 \mathrm{hPa}$ to the surface; (bottom) as in top but averaged over $700 \mathrm{hPa}$ to the surface. All are averaged over (left) $34^{\circ}$ to $37^{\circ}$ and (right) $54^{\circ}$ to $57^{\circ}$ latitude.

eddy momentum flux convergence that is not balanced by the surface stress associated with the anomalous zonal wind at the beginning of the spinup. This creates a positive momentum budget residual that acts to accelerate the zonal wind. Changes of the opposite sign are seen in the latitudes of deceleration on the equatorward side of the jet. Equatorward of $\sim 30^{\circ}$ latitude, the changes in eddy momentum flux are smaller (particularly at the beginning of the spinup), resulting in a more complex balance in which the momentum flux due to the zonally averaged circulation is also important. During the spinup, the zonal wind anomalies extend down to the surface and the anomalous surface stress increasingly balances the anomalous eddy forcing so that, by equilibrium (bottom panels of Fig. 4), there is almost a complete balance.

The vertically integrated momentum budget therefore confirms that the altered eddy momentum flux is important in driving the vertically integrated zonal wind accelerations. This analysis has been shown for the conventional Eulerian mean (CEM), but the transformed Eulerian mean (TEM) momentum budget has also been studied. In the vertical integral the two formulations are equivalent, but the local TEM balances in the meridional plane are quite different for the E5 spinup. The change in Eliassen-Palm (E-P) flux divergence has a very broad latitudinal structure that is closely balanced by the residual circulation term $\left(f-\bar{u}_{y}\right) \bar{v}^{*}$ and neither corresponds 

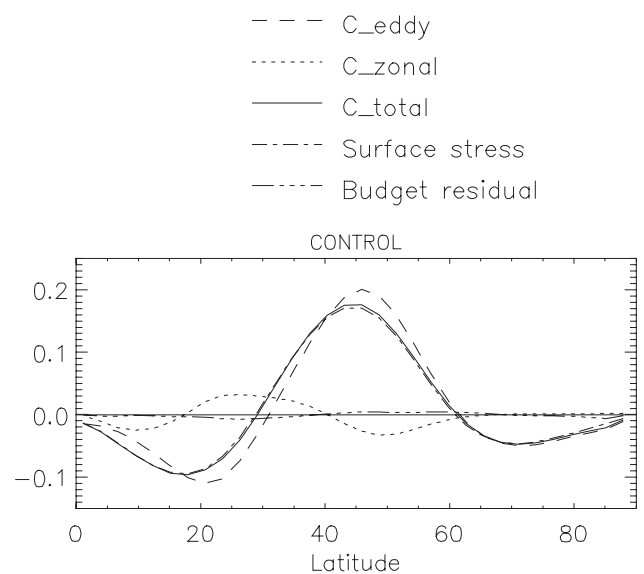

DAYS O TO 9 - CONTROL

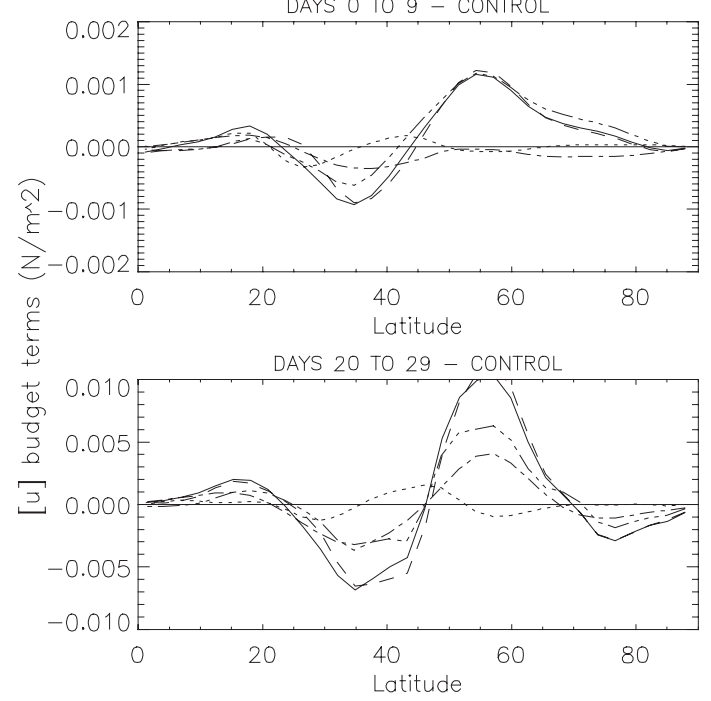

E5 - CONTROL

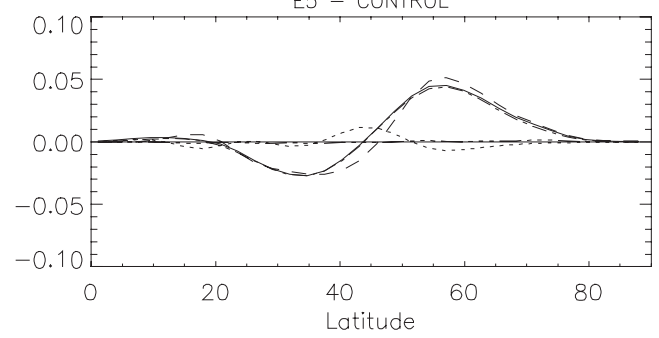

FIG. 4. Vertically integrated momentum budget. (top) Control run vertically integrated momentum budget; (bottom) difference between the vertically integrated momentum budget of the equilibrium E5 run and the control run; (middle) difference between the vertically integrated momentum budget for days 0 to 9 and 20 to 29 of the $\mathrm{E} 5$ run and the vertically integrated momentum budget of the equivalent 10-day chunks of the control run. Note the different scales on the ordinate axes. The convergence of horizontal eddy momentum flux, convergence of momentum flux by the zonally averaged flow, and their sum $\left(C_{\text {total }}\right)$ are shown along with the anomalous surface stress and the budget residual. Positive surface stress has been taken as corresponding to an easterly forcing so the surface stress is taken away from each of the other terms to obtain a balance. to the latitudinal structure of the changes in zonal wind $\bar{u}$. This broad structure is dominated by the vertical E-P flux component in the early spinup, as shown in Fig. 2b. The TEM version of the momentum budget therefore does not aid interpretation of the results.

\section{c. What drives the changes in eddy momentum flux?}

Changes in eddy momentum flux around the tropopause region and in the upper troposphere appear to be pivotal in producing the tropospheric response to the stratospheric heating perturbation. In this section we discuss what gives rise to this altered eddy momentum flux.

Figure $2 b$ shows the evolution of the change in E-P flux throughout the E5 spinup, where the arrows have been scaled for graphical purposes following the conventions of Edmon et al. (1980). Recall that in the quasigeostrophic approximation, $\bar{F}=\left(F_{\phi}, F_{p}\right)$, where $F_{\phi}=-a \cos \phi\left(\overline{u^{\prime} v^{\prime}}\right)$ and $F_{p}=a f \cos \phi\left(\overline{v^{\prime} \theta^{\prime}} / \overline{\theta_{p}}\right)$. When eddy propagation can be thought of as wavelike, then the direction of the E-P flux also indicates the direction of wave propagation. In the equilibrium situation for the control run (top panel) eddies develop because of baroclinic instability of the temperature gradient below the jet and propagate upward along the jet axis toward the high static stability of the tropopause. This prevents further upward propagation and the eddies refract primarily equatorward to break anticyclonically on the equatorward side of the jet. The equatorward propagation corresponds to the region of poleward eddy momentum flux in the top panel of Fig. 2a.

Even at the beginning of the spinup (days 0 to 9) there are changes to the direction of eddy propagation around the tropopause, with a weakening of the upward E-P flux particularly at lower latitudes (i.e., equatorward of the jet maximum). This is accompanied by reduced equatorward E-P flux around the tropopause $(\sim 100$ to $250 \mathrm{hPa}$ ) equatorward of $\sim 40^{\circ}$, corresponding to the decrease in horizontal eddy momentum flux seen on the equatorward side of the jet. Below this, in the upper troposphere, there is increased equatorward propagation associated with the increased horizontal eddy momentum flux that extends equatorward and downward from the poleward side of the jet. The weakened upward E-P flux extends down to the surface by days 20 to 29 , and by days 40 to 49 (not shown) it is accompanied by increased upward E-P flux on the poleward side of the jet, consistent with the shift in the region of maximum baroclinicity with the shift in the jet. This is further amplified at equilibrium (bottom panel).

To determine whether the changes in E-P flux throughout the spinup are consistent with changes in wave refraction by the evolving zonal-mean state, the zonal-mean quasigeostrophic refractive index (Matsuno 1970) has 
been computed. For a dimensionless zonal wavenumber $k$, this is given by

$$
n^{2}=\left[\frac{\bar{q}_{\phi}}{a(\bar{u}-c)}-\left(\frac{k}{a \cos \phi}\right)^{2}-\left(\frac{f}{2 N H}\right)^{2}\right] a^{2},
$$

where $c$ is the zonal phase speed, $N$ is the buoyancy frequency, and $H$ is the density scale height.

The meridional gradient of potential vorticity $\bar{q}_{\phi}$ is given by

$$
\bar{q}_{\phi}=2 \Omega \cos (\phi)-\left[\frac{(\bar{u} \cos \phi)_{\phi}}{a \cos \phi}\right]_{\phi}+\frac{a f^{2}}{R_{d}}\left(\frac{p \theta}{T} \frac{\overline{u_{p}}}{\overline{\theta_{p}}}\right)_{p} .
$$

Karoly and Hoskins (1982) demonstrate that under linear Wentzel-Kramers-Brillouin (WKB) theory, waves will be refracted by gradients of $n^{2}$ such that they will tend to propagate away from regions of low refractive index and toward regions of high refractive index.

The top panel of Fig. 5 shows the change in refractive index together with the change in E-P flux for days 0 to 9 of the spinup for the upper troposphere/tropopause region around the jet latitude, where the change in E-P flux is most significant. When calculating differences in the refractive index, the term involving wavenumber cancels out for any particular wavenumber. The refractive index calculations have assumed a phase speed of $8 \mathrm{~m} \mathrm{~s}^{-1}$. The following discussion of refractive index and E-P flux anomalies applies to wavenumbers 5-7, which dominate the E-P flux and horizontal eddy momentum flux anomalies and also dominate in the calculation of the phase speed.

It can be seen that the changes in E-P flux are generally consistent with those in the refractive index. There is a vertical dipole change in refractive index consisting of a reduction around the tropopause and an increase below it (i.e., a reduced upward gradient of

\footnotetext{
${ }^{2}$ The phase speed was estimated by tracking regions of maximum potential vorticity around the jet latitude (between $40^{\circ}$ and $50^{\circ}$ latitude). Calculation of the gradients of best-fitting lines joining points of maximum potential vorticity in the longitude-time plane gives a distribution of phase speeds centered on $\sim 8 \pm 2 \mathrm{~m} \mathrm{~s}^{-1}$. The patterns of change in refractive index over the spinup are qualitatively similar for any choice of phase speed between 6 and $16 \mathrm{~m} \mathrm{~s}^{-1}$. There was no detectable shift in the mean phase speed over the spinups. This is in contrast with the study by Chen et al. (2007), which showed that a poleward shift of the midlatitude jets in response to a reduction in surface friction was due to an increase in the phase speed of the eddies and therefore a poleward shift of the subtropical critical latitude. However, our experiments are fundamentally different in not imposing a change in zonal wind in the troposphere. The results of Chen et al. (2007) occur because eddies grow on a strengthened jet when friction is reduced: no such systematic change occurs in our spinup experiments.
}
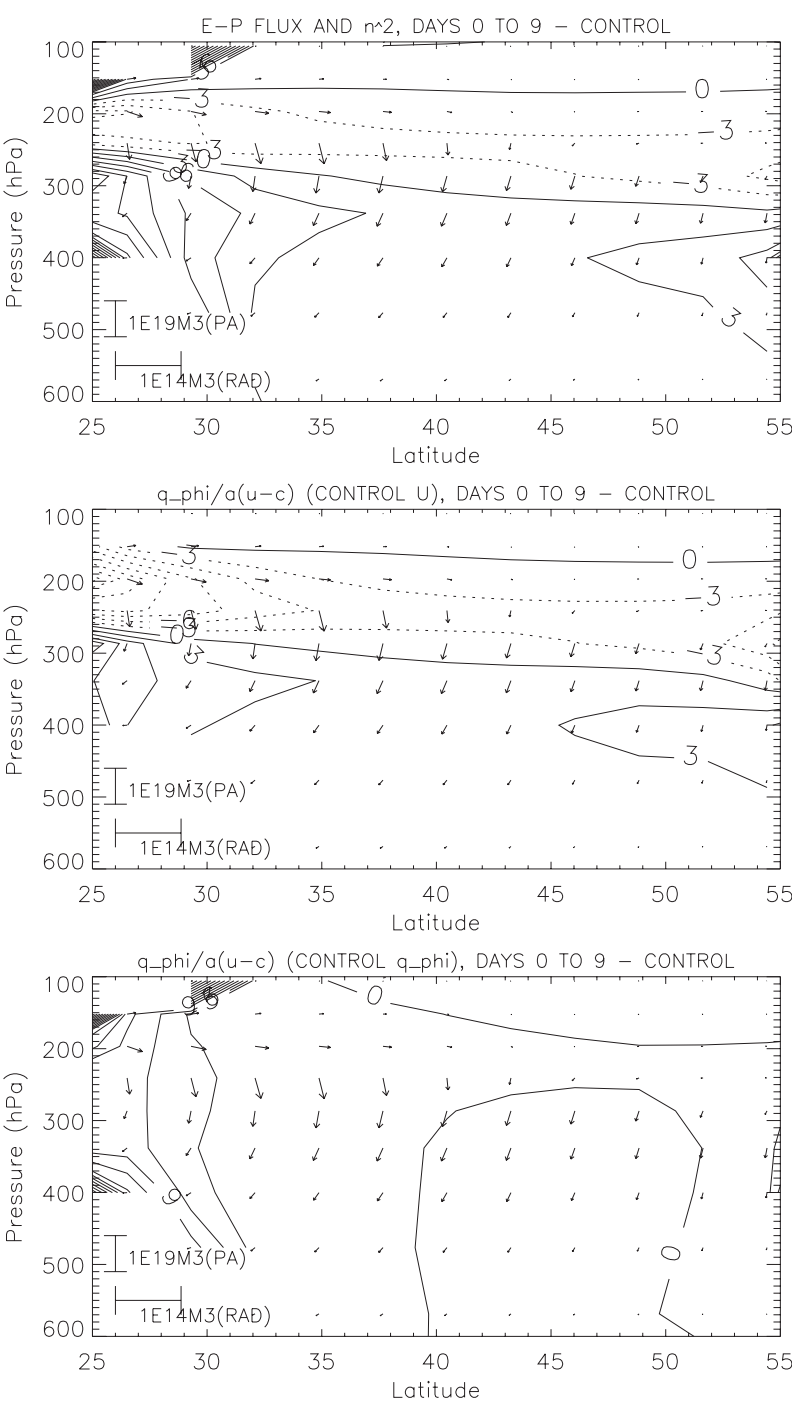

FIG. 5. (top) Change in $n^{2}$ (contours) and scaled E-P flux (arrows) for days 0 to 9 of the E5 spinup; (middle) change in $\bar{q}_{\phi} / a(\bar{u}-c)$ using the change in $\bar{q}_{\phi}$ from the spinup and $u$ from the control run; (bottom) changes in $\bar{q}_{\phi} / a(\bar{u}-c)$ using changes in $\bar{u}$ from the spinup and $\bar{q}_{\phi}$ from the control run. Contours have been blanked out in regions where $c>u$. Note that values in the middle and bottom panels have been scaled by $a^{2}$ to make them nondimensional for comparison with total $n^{2}$.

refractive index, with the refractive index changes being larger on the flanks of the jet). The reduced upward gradient of $n^{2}$ is accompanied by reduced upward E-P flux, with the anomalies being larger on the equatorward side of the jet (particularly at $\sim 300 \mathrm{hPa}$ ). By continuity, this reduced upward E-P flux would be expected to be accompanied by a reduced equatorward E-P flux above, as is indeed the case. This can be seen to be consistent with refraction away from a minimum in $n^{2}$ in low latitudes at around $200 \mathrm{hPa}$. Thus, the initial weakening of the upward E-P flux (and associated 
change in horizontal E-P flux above) appears to stem from the decrease in refractive index around the tropopause and the increase below, which reduces the upward gradient of refractive index and thus weakens upward eddy propagation. Part of this change in vertical E-P flux is associated with a change in horizontal eddy heat flux and part of it is due directly to the change in the vertical gradient of potential temperature that is within the definition of the vertical E-P flux component.

The individual contributions to the change in $n^{2}$ in Eq. (4) have been examined to determine what aspects of the change in the zonal mean state lead to the changes in wave propagation. The change in $n^{2}$ is calculated for a given wavenumber so the middle term of Eq. (4) is fixed. Comparison of the other two terms show that the change in the third term is insignificant compared to the change in $\bar{q}_{\phi} / a(\bar{u}-c)$. The lower two panels of Fig. 5 compare the effect on $\bar{q}_{\phi} / a(\bar{u}-c)$ of changes in $\bar{q}_{\phi}$ and of changes in $\bar{u}$ in the denominator. The middle panel shows the effect of the change in $\bar{q}_{\phi}$ only, by calculating the change in $\bar{q}_{\phi} / a(\bar{u}-c)$ using the spinup values of $\bar{q}_{\phi}$ and the control run values of $\bar{u}$ in the denominator. Conversely, the bottom panel shows the change in $\bar{q}_{\phi} / a(\bar{u}-c)$ using the spinup value of $\bar{u}$ in the denominator and the control run value of $\bar{q}_{\phi}$.

Comparison with the top panel of Fig. 5 shows that most of the refractive index change is explained by the change in $\bar{q}_{\phi}$, except at the lowest latitudes. The contribution due to the change in $\bar{u}$ in the denominator is small and confined to the low-latitude tropopause region.

Initially, it is the change in the meridional gradient of potential vorticity at the tropopause that alters the refractive index and thus eddy propagation. The top panel of Fig. 6 shows the change in meridional PV gradient for days 0 to 9 , which consists of this dipole change with a decrease around the tropopause and an increase below. Comparing this with the change in E-P flux (Fig. 2b), it can be seen that the reduced upward E-P flux only occurs in the latitudes where the dipole change in $\bar{q}_{\phi}$ occurs. The components of $\bar{q}_{\phi}$ are next diagnosed to understand how this change in $\bar{q}_{\phi}$ arises. Equation (5) gives the meridional gradient of quasigeostrophic potential vorticity as a function of the zonal-mean zonal wind $(\bar{u})$ and potential temperature $(\bar{\theta})$ gradients. The second term measures the meridional curvature of the zonal wind and augments the planetary vorticity gradient. The third term is influenced by changes in the vertical shear and curvature of the zonal wind and the vertical shear and curvature of the potential temperature.

The change in the third term,

$$
\frac{a f^{2}}{R_{d}}\left(\frac{p \bar{\theta}}{\bar{T}} \frac{\bar{u}_{p}}{\bar{\theta}_{p}}\right)_{p},
$$
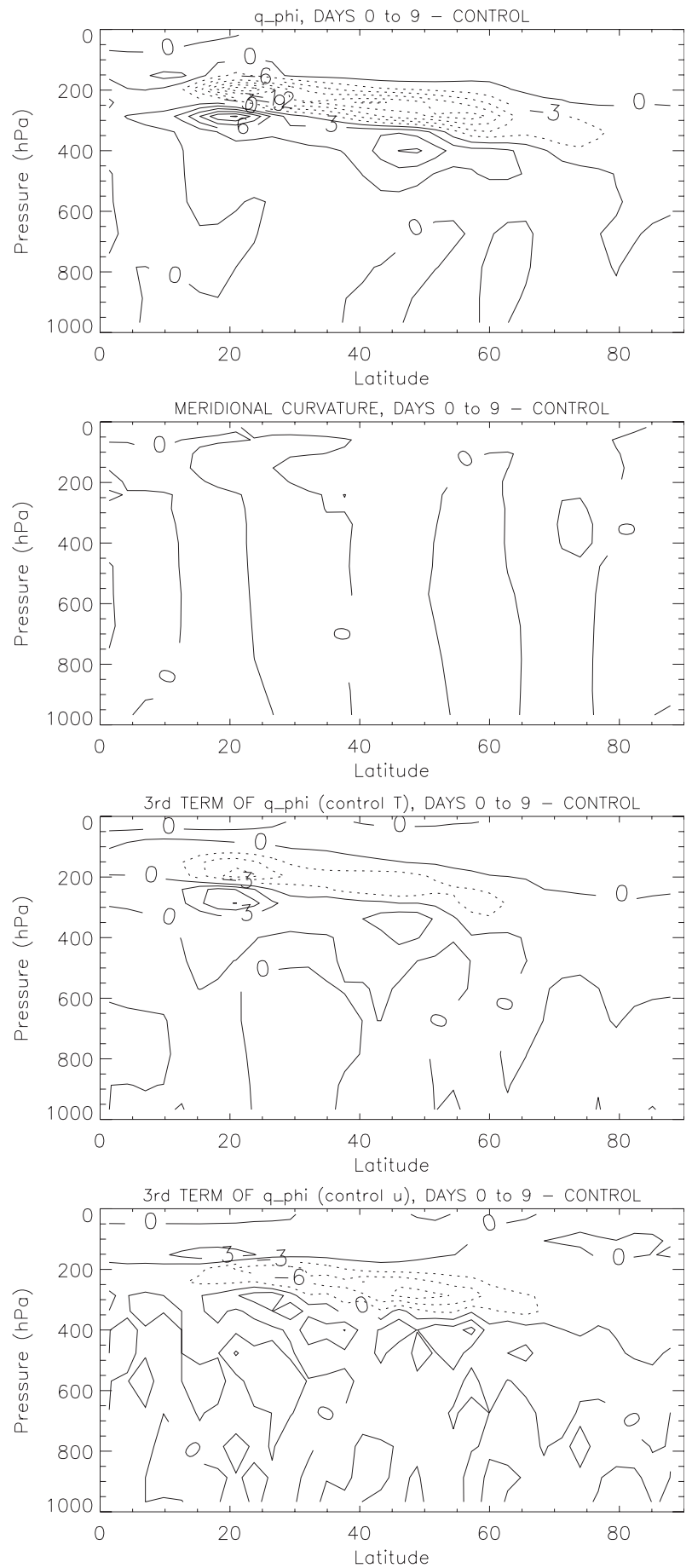

FIG. 6. Change in individual components of $\bar{q}_{\phi}\left(10^{-6} \mathrm{~s}^{-1} \mathrm{rad}^{-1}\right)$. (top) Change in $\bar{q}_{\phi}$; (upper middle) meridional curvature; (bottom middle) change in third term of $\bar{q}_{\phi}$ due to altered $\bar{u}$; (bottom) change in third term of $\bar{q}_{\phi}$ due to altered $\bar{\theta}$. Contour interval $=3 \times$ $10^{-6} \mathrm{~s}^{-1} \mathrm{rad}^{-1}$. 
can be linearized in terms of the contributions from the change in $\bar{\theta}$ and the change in $\bar{u}$ where the nonlinear part is small.

The lower three panels of Fig. 6 show the separate contributions to the change in meridional PV gradient for days 0 to 9 of the E5 spinup. The contribution to the change from the meridional curvature is negligible compared to the contributions from the other terms. The contributions to the change in term 6 from the changes in $\bar{u}$ and $\bar{\theta}$, shown in the third and fourth panels respectively, are both important, with the change due to the vertical temperature gradient being about twice as large as that due to vertical zonal wind gradients (or equivalently horizontal temperature gradients), except perhaps at low latitudes. These both act to give this dipole change in meridional PV gradient, which then results in the dipole change in $n^{2}$. The $n^{2}$ anomalies on the flanks of the jet are amplified by the effect of low values of $(\bar{u}-c)$ in the denominator. This initial change in $n^{2}$ produces refraction of the E-P flux of the form shown in Fig. 2b, which results in changes in horizontal eddy momentum flux. This then drives zonal wind anomalies in the upper troposphere as well as anomalous meridional circulations, which results in zonal wind and temperature changes in the lower troposphere.

There is, however, an important feedback of the initial zonal wind changes on the eddies that is apparent in Fig. 7 (equivalent to Fig. 5, but now for days 40 to 49). The refractive index and E-P flux changes are now considerably larger and there is a positive gradient of refractive index from pole to equator in the troposphere accompanied by anomalous equatorward refraction of E-P flux in the troposphere. This leads to the increase in horizontal eddy momentum flux stretching down into the troposphere seen in Fig. 2a. Comparing the total change in refractive index with the individual components demonstrates that the change in $\bar{q}_{\phi}$ no longer completely explains the change in $n^{2}$. There is now a much larger contribution from the altered zonal wind in the denominator. As the zonal wind in the troposphere starts to change, the $\bar{u}$ and $\bar{q}_{\phi}$ contributions become comparable. In the region of zonal wind deceleration between $\sim 30^{\circ}$ and $45^{\circ}$ the zonal wind (in the denominator) contribution to $\bar{q}_{\phi} / a(\bar{u}-c)$ actually dominates the refractive index changes in the troposphere by days 10 to 19 . However, the change in PV gradient remains the dominant contribution to the change in $n^{2}$ around the tropopause. The lower panel of Fig. 7 demonstrates that the altered zonal wind is responsible for the positive gradient of refractive index from pole to equator in the troposphere and corresponding E-P flux and eddy momentum flux changes in the troposphere. These results suggest a feedback: as the zonal wind starts to respond
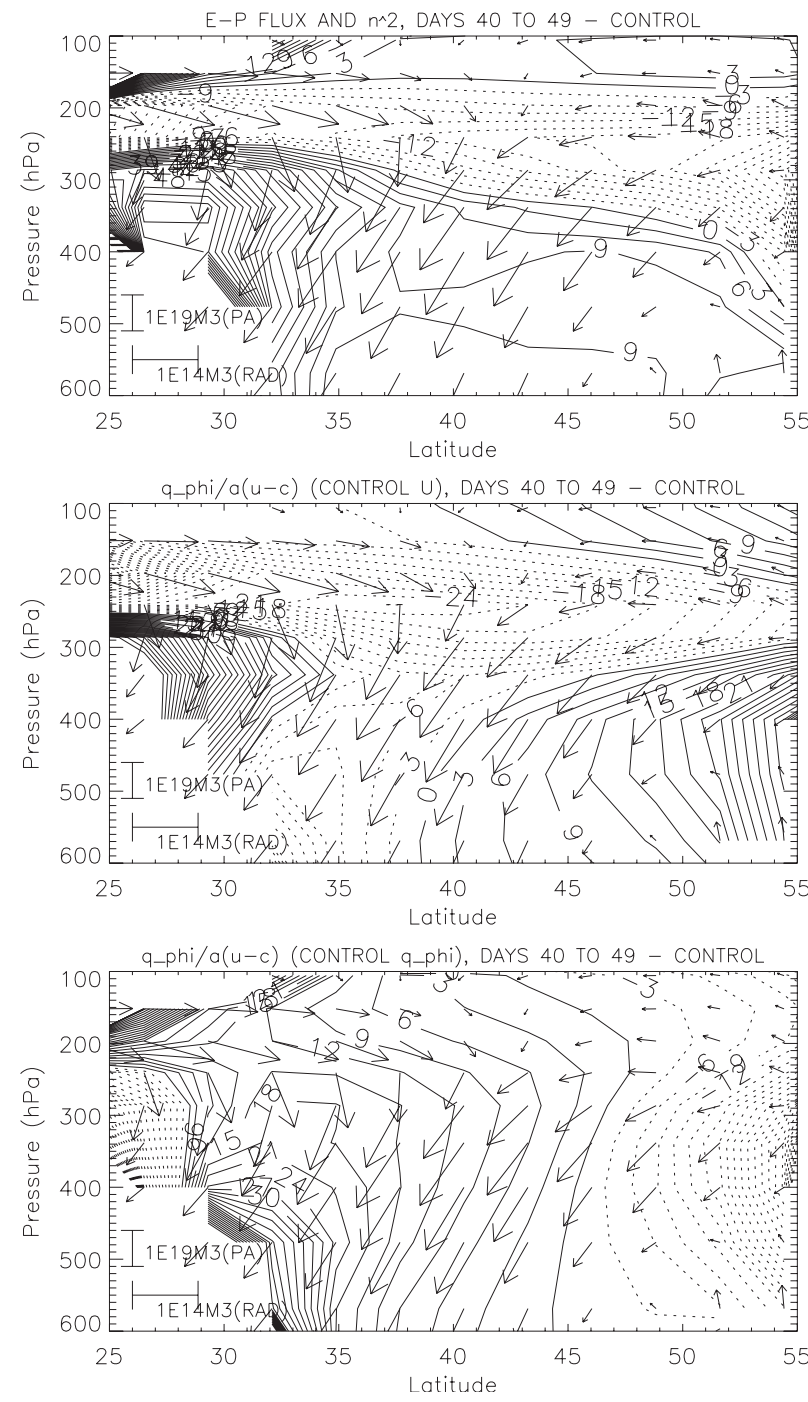

FIG. 7. As in Fig. 5, but for days 40 to 49.

to the initial changes in meridional temperature gradient and eddy momentum flux around the tropopause, this influences eddy propagation in the troposphere, resulting in changes in horizontal eddy momentum flux throughout the troposphere. This acts to further accelerate the tropospheric zonal wind. The initial response followed by a feedback involving the tropospheric eddies is likely to be the reason for the two stage response seen in Fig. 3: as the feedback becomes important, there is a stronger acceleration of the zonal wind.

\section{Comparison with a zonally symmetric model}

The results of section 3 have demonstrated the importance of changing eddy momentum fluxes in the tropospheric response to the applied heating perturbation. In this section we compare these results with those for a 

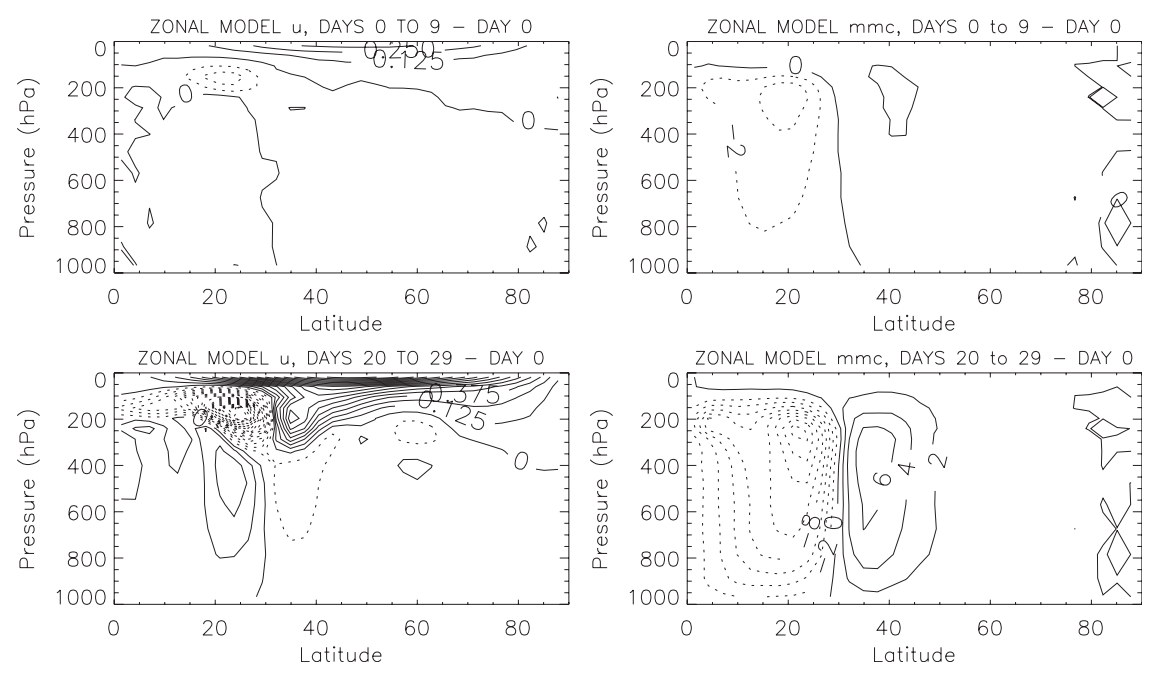

FIG. 8. (left) Zonal-mean zonal wind and (right) mean meridional circulation $\left(10^{8} \mathrm{~kg} \mathrm{~s}^{-1}\right)$ evolution over the zonal E5 spinup. (top) Days 0-9; (bottom) days 20-29. The zonal wind contour intervals are the same as in Fig. 1, but the mean meridional circulation contour interval is halved.

zonally symmetric model in which the same equatorial heating perturbation as in section 3 is applied but the eddy fluxes are held fixed at the values required to maintain the control run equilibrium state. Because of the lack of eddy feedback in the zonally symmetric model only one run was needed for this spinup experiment.

Figure 8 shows the spinup evolution of the streamfunction of the mean meridional circulation and the zonal-mean zonal wind for the zonal model E5 case. Comparison with Fig. 1 demonstrates that initially the zonal wind response in the stratosphere is rather similar to the full model. The meridional circulation changes in the zonal model at days 0 to 9 are, however, considerably reduced. There is a weak anomalous indirect circulation at low latitudes and a direct circulation beyond around $30^{\circ}$ latitude. This is the symmetric circulation response to maintain thermal wind balance in the presence of the altered meridional temperature gradients. Coriolis torques on these anomalous meridional circulations drive changes in the zonal wind. However, the changes in horizontal eddy momentum flux convergence that enhanced these circulation anomalies in the 3D model are absent in the zonal model.

After days 0 to 9 , the tropospheric circulation responds differently. There is a large response in the zonal wind in the subtropical upper troposphere of the zonally symmetric model, associated with a large temperature increase that stretches down into the troposphere in the subtropics. No such temperature increase is present in the $3 \mathrm{D}$ model. In the zonal model, examination of the terms in the thermodynamic equation (not shown) demonstrates that this tropospheric temperature increase is due to vertical advection: the meridional circulation advects the stratospheric temperature increase down into the subtropical troposphere. This does not occur in the $3 \mathrm{D}$ model runs because between $\sim 15^{\circ}$ and $30^{\circ}$ latitude there is a stronger anomalous upward vertical motion throughout the spinup that counteracts it.

It is clear that in the zonal model the initial stratospheric response is rather similar to that in the 3D model, but the tropospheric response is far weaker. There is no weakening and poleward shift of the midlatitude jets. Thus, most of the response in the troposphere of the 3D model is due to altered eddy fluxes.

Figure 9 shows the refractive index change for days 0 to 9 of the zonal model E5 spinup (analogous to that in Fig. 5). This can be thought of as showing the initial change in refractive index that influences the eddies before the resulting changes in the eddies alter the circulation (and consequently the refractive index). The initial reduction in $n^{2}$ at the tropopause and increase below it, seen in the 3D model, also occurs in the zonal model, but comparison of Figs. 5 and 9 also shows that the eddies have started to alter the circulation in the 3D model even in the initial 10-day period. In the zonal model, the reduced vertical gradient in $n^{2}$ is much larger in the low latitudes where the reduced upward E-P flux in the 3D model is strongest. This is particularly true of the contribution due to the negative part of the dipole in $n^{2}$.

The initial changes that alter the refractive index in the stratosphere and around the tropopause are therefore a direct response to the imposed heating, but the full tropospheric response seen in the 3D model requires there to be changes in the eddies. 


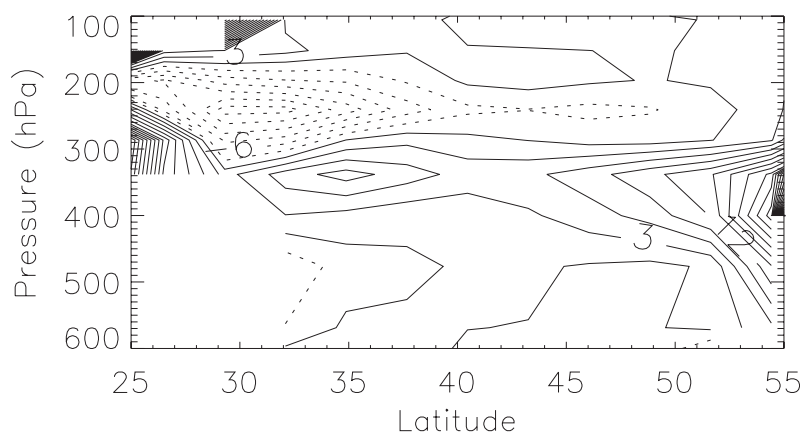

FIG. 9. Change in $n^{2}$ at days 0 to 9 for the zonal model E5 case.

\section{The U5 and P10 experiments}

To confirm that the above analysis is also valid for other stratospheric heating perturbations, we briefly compare with the results of the U5 and P10 experiments, focusing first on the initial changes in the meridional PV gradient and eddy refraction and then demonstrating that the feedback involving tropospheric zonal wind changes also occurs in these experiments. We also seek to understand what determines the direction of jet displacement because in both these experiments the jet is displaced equatorward (HBD05), as opposed to poleward in E5.

The changes in $\bar{q}_{\phi}$, E-P flux, and horizontal eddy momentum flux for days 0 to 9 of the U5 and P10 spinups in the 3D model are shown in Fig. 10. It was demonstrated in section 3 that the change in vertical temperature gradient around the tropopause was important in the initial change in $\bar{q}_{\phi}$. The top panels of Figs. 10 and 6 clearly show that the differences in the $\bar{q}_{\phi}$ anomaly between the experiments are associated with differences in the location of the change in vertical temperature gradient. In U5 the stratosphere is heated uniformly at all latitudes. As a result, the vertical temperature gradient changes at all latitudes, and the anomalous $\bar{q}_{\phi}$ has a greater meridional extent than in E5. In P10, the changes in vertical temperature gradient and $\bar{q}_{\phi}$ are largest in the polar region, whereas in E5 the changes are largest at lower latitudes.

The middle panels of Fig. 10 demonstrate that the reduced upward E-P flux occurs in the regions where the PV gradient is most changed. Therefore, U5 shows a more uniform decrease in upward E-P flux than either $\mathrm{P} 10$ or E5, in which the change is largest at high or low latitudes, respectively. The spatial correspondence between the reduction in vertical E-P flux and change in $\bar{q}_{\phi}$ arises through both the change in refractive index and the direct dependence of vertical E-P flux on static stability.

The bottom panels of Fig. 10 show the corresponding differences in the horizontal eddy momentum flux anom- alies. In U5 there is simply a weakening of the control horizontal eddy momentum flux (Fig. 2a) around the tropopause (but with a slight equatorward displacement of the zero line), whereas for P10 the anomalies are larger in higher latitudes.

The different locations of anomalous horizontal eddy momentum flux in each experiment lead to different latitudinal extents of the anomalous momentum flux convergence and hence of initial zonal wind acceleration/ deceleration at the tropopause. This is spread downward by the meridional circulation and generates correspondingly different feedbacks on the eddies.

This can be seen in Fig. 11, which shows the zonal wind together with $n^{2}$ and E-P flux anomalies for days 40 to 49 of the U5 and P10 experiments. In P10 the zonal wind decreases on the poleward side of the jet and increases on the equatorward side. By arguments analogous to those for E5, this results in a positive $n^{2}$ gradient anomaly from low latitudes to high latitudes that is accompanied by a poleward refraction of E-P flux and corresponding decrease in horizontal eddy momentum flux in the troposphere, which further acts to reinforce these zonal wind anomalies.

The feedback is less apparent in U5 because the zonal wind anomalies do not yet dominate over the PV gradient changes in the subtropical upper tropospheric $n^{2}$, so there is not a positive $n^{2}$ gradient toward the pole throughout the whole of the troposphere. However, some poleward refraction can be seen toward the maximum in $n^{2}$ on the poleward side of the jet in the troposphere, associated with the zonal wind decrease there.

Comparison of these different experiments demonstrates that it is the change in vertical temperature gradient and how it is localized in latitude that determine where the eddy momentum flux changes initially relative to the jet. This determines how the zonal flow begins to evolve and the subsequent direction of the jet displacement.

\section{Discussion and conclusions}

Our analysis of the model results, described above, leads us to propose the following mechanism, summarized in Fig. 12, whereby thermal perturbations to the stratosphere influence the tropospheric circulation.

Heating the stratosphere causes both the vertical and meridional temperature gradients around the tropopause region to change. Changes in vertical shear and curvature of the zonal wind, associated with the change in meridional temperature gradient, alter the meridional PV gradient around the tropopause. Perhaps more importantly, the change in vertical temperature gradient and curvature has a direct effect on the meridional 

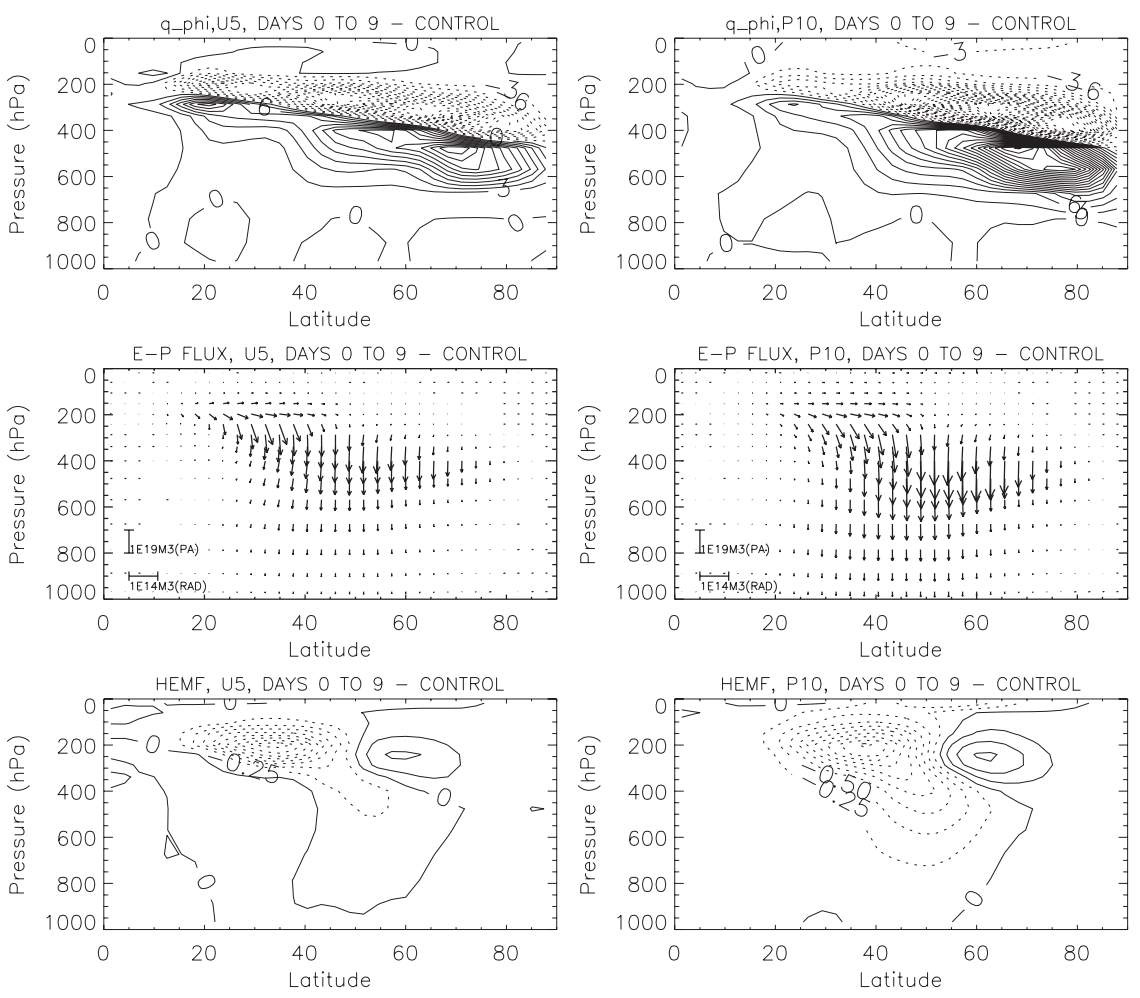

(a) $\cup 5$

(b) $\mathrm{P} 10$

FIG. 10. (top) Change in $\bar{q}_{\phi}\left(10^{-6} \mathrm{~s}^{-1} \mathrm{rad}^{-1}\right)$; (middle) change in E-P flux; (bottom) change in horizontal eddy momentum flux, with plots for days 0 to 9 of the (a) U5 and (b) P10 case.

PV gradient. This produces a change in refractive index that influences the direction of propagation of the eddies, creating horizontal eddy momentum flux anomalies and heat flux anomalies. The changes in eddy momentum flux act to drive changes in the zonal wind locally around the tropopause and in the upper troposphere. They also drive anomalous meridional circulations that result in zonal wind and temperature changes throughout the troposphere. These tropospheric zonal wind changes alter the refractive index in a local positive feedback as follows: Reduced zonal wind increases the ambient positive refractive index because the term $(\bar{u}-c)$ appears in the denominator of $n^{2}$; then wave activity is refracted toward that latitude, thus increasing E-P flux convergence, which drives further easterly acceleration (and vice versa for westerly anomalies). The spreading of initial zonal wind anomalies throughout the depth of the troposphere also creates an implicit feedback. An easterly anomaly with easterly vertical shear reduces the local baroclinicity: this weakens the E-P flux source and thus weakens eddy forcing of westerly flow at that latitude. Both of these mechanisms cause the eddies to provide a positive feedback on displacements of the midlatitude jet.
There has been some debate as to whether the refractive index can be used to predict wave behavior when the waves are not strictly in the WKB limit. However, several authors have demonstrated that the refractive index can give useful predictions as to the behavior of waves even when the WKB conditions do not strictly apply (Chen and Robinson 1992; Hartmann and Zuercher 1998; Lorenz and Hartmann 2003). Moreover, it has been demonstrated in Figs. 5 and 7 that the refractive index in our results is consistent with the altered E-P flux and so is useful in predicting the changes in eddy propagation in the midlatitude tropopause region.

The importance of changes in eddy momentum flux around the tropopause region in driving the tropospheric response has been demonstrated by the 3D spinup experiments and has been confirmed by the lack of response in the troposphere of the zonally symmetric model with fixed eddy forcing. The requirement for a feedback involving the tropospheric eddy momentum fluxes to produce the full zonal wind anomalies is in agreement with several previous studies (Polvani and Kushner 2002; Kushner and Polvani 2004, 2006; Song and Robinson 2004). The idea of tropospheric eddy 

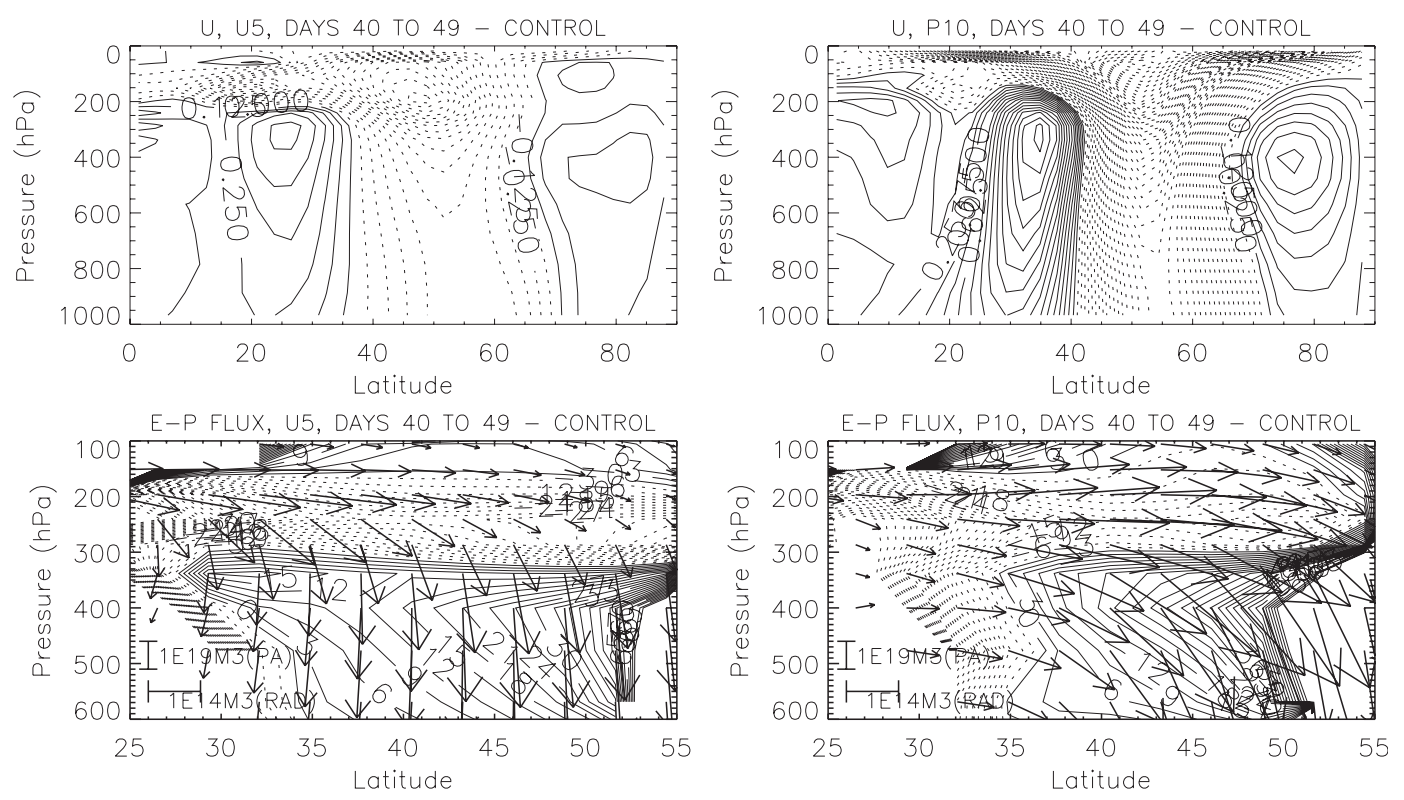

FIG. 11. (top) Change in $\bar{u}$ for days 40 to 49 of the (left) U5 and (right) P10 spinups; (bottom) $n^{2}$ and E-P flux anomalies for the same time zoomed in around the midlatitude upper troposphere-tropopause region.

feedback has been developed further in this study by using the refractive index to show that as the zonal wind changes in the troposphere, it results in meridional gradients of refractive index, which in turn result in eddy refraction in the troposphere to produce changes in tropospheric eddy momentum fluxes.

The model used here differs from some previous studies, such as those of Kushner and Polvani (2004, 2006), in that it does not have a stratospheric polar vortex but still produces a significant tropospheric response to stratospheric heating perturbations. Furthermore, because there is no large-scale zonally asymmetric forcing in our model, planetary waves are weak and eddy forced: the dominant wavenumbers are 5, 6, and 7. Our results therefore confirm the possibility that smaller-scale baroclinic eddies alone can produce a tropospheric response to stratospheric heating perturbations, although larger-scale planetary waves may play a role in other modeling studies and in the real atmosphere. A mechanism by which a tropospheric response can be produced by smaller-scale baroclinic eddies is also consistent with observed signals that are symmetric about the equator and/or seen in all seasons, such as the zonal wind and temperature response observed over the solar cycle (Haigh 2003; HBD05).

Moreover, our results suggest that it is a change in eddy propagation near the eddy source latitudes that is important in producing the jet displacement rather than processes affecting the critical latitude of eddy breaking in the subtropics. Following the results of Chen et al.
(2007), who showed the importance of a change in phase speed in the poleward shift of midlatitude westerlies in response to a change in surface friction, Chen and Held (2007) have suggested that a similar mechanism could produce a tropospheric response to stratospheric zonal wind anomalies. By this hypothesis, changes in lower stratospheric and upper tropospheric zonal wind could produce a shift in the midlatitude jets through a shift in the region of subtropical wave breaking caused by a change in phase speed. However, Chen and Held (2007)

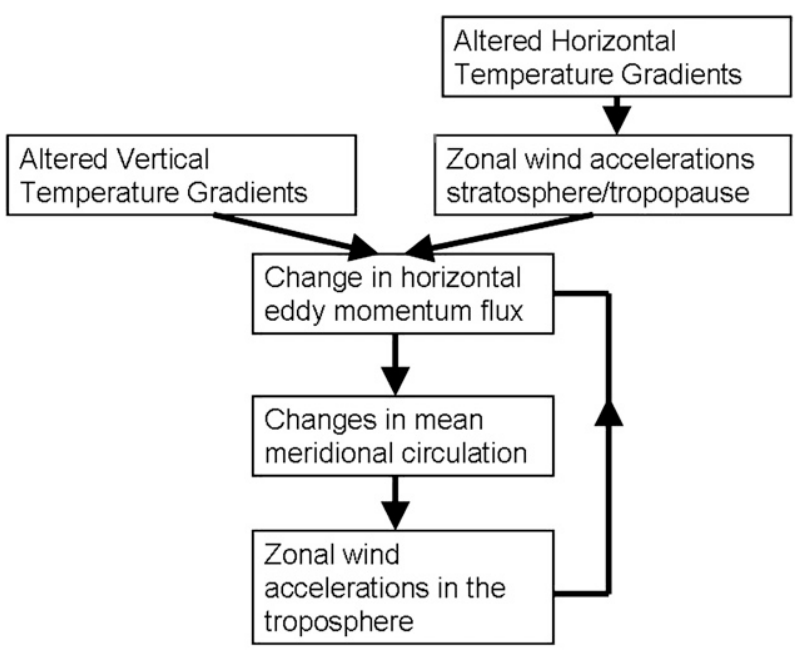

FIG. 12. Summary of the mechanism by which the heating perturbation in the stratosphere is influencing tropospheric circulation. 
state that the question still remains as to whether the shift in the phase speeds is a consequence or a cause of the tropospheric circulation changes. In our experiments, a change in phase speed shifting the critical line does not appear to be an important factor.

Our primary aim was to study how the response to changing solar activity could be produced in the troposphere, but our results equally apply to other situations in which there is a heating or cooling perturbation applied to the stratosphere. For example, Lorenz and DeWeaver (2007) showed, in model studies investigating the response to stratospheric cooling, that a complete understanding of the mechanisms involved must consider both the effects of the change in vertical temperature gradient and the change in horizontal temperature gradient. Our results have confirmed this: the change in horizontal temperature gradient is important through its influence on the zonal wind shear and the subsequent effect on the eddies. However, the changes in vertical temperature shear and curvature around the tropopause, and how they are localized in latitude, are key to the latitudinal distribution of the tropospheric response and therefore to the direction of meridional jet displacement.

Gerber and Vallis (2007) and Gerber et al. (2008) have shown that simplified models such as ours can have unrealistically long decorrelation times compared to the real atmosphere. This can lead such models to be overly sensitive to external forcing, as implied by the fluctuation-dissipation theorem (Leith 1975). The sensitivity of these model results to vertical resolution and the introduction of zonal asymmetries is elaborated upon in the appendix. We do find there is a sensitivity in the magnitude of the response to these factors, but the results are qualitatively the same and our conclusions about the mechanisms involved in producing the tropospheric response remain valid.

To summarize: HBD05 demonstrated that tropospheric circulation changes, similar in nature to those seen over the solar cycle, can be produced by heating the stratosphere of a simplified GCM preferentially in the equatorial region. Here we have investigated the mechanisms by which such a response is produced and have demonstrated the importance of changes in eddy momentum fluxes in driving the tropospheric circulation changes and of the presence of tropospheric eddy feedbacks on the zonal wind. The quasigeostrophic refractive index has demonstrated the initial importance of altered vertical temperature gradient and vertical wind shear in changing the eddy momentum flux. Furthermore, it shows that it is the zonal wind anomalies in the troposphere that refract the eddies there to provide the feedback.

Some analyses of zonal mean temperatures (Crooks and Gray 2005) suggest that at solar maximum the lower stratospheric heating is largest in lobes that extend into the subtropics. This might enhance the tropospheric response because the heating maxima are closer to the latitudes of maximum eddy activity. Further work will address the sensitivity of the response to details of the distribution of stratospheric heating.

Acknowledgments. Isla Simpson was funded by a UK Natural Environment Research council PhD studentship. We thank John Methven and Sarah Sparrow for useful discussions. We are also grateful to three anonymous reviewers for their helpful comments.

\section{APPENDIX}

\section{Sensitivity to Model Resolution and Topography}

Gerber and Vallis (2007) and Gerber et al. (2008) have shown that the decorrelation time scale of the leading mode of annular variability in sGCMs is sensitive to both the model resolution and the introduction of zonally asymmetric boundary conditions such as topography. Without such zonally asymmetric boundary conditions, the decorrelation time scales are known to be unrealistically long, which the fluctuation-dissipation theorem implies can lead to these models being overly sensitive to external forcing. Here we present some initial tests of the sensitivity of the model results to increased vertical resolution and zonally asymmetric boundary conditions.

Figure A1 shows the equilibrium response to E5 heating for a model run with T42L30 resolution and a run with the original T42L15 resolution but with the introduction of topography. These are produced from the difference between a 5000-day equilibrated E5 run and a 5000-day control run. Results for the T42L30 run have been averaged over both hemispheres as previously.

Focusing first on the T42L30 run, the center of the midlatitude jet in the control run is further equatorward at around $40^{\circ}$ latitude. Figure A1a clearly shows a qualitatively similar zonal wind response to the E5 heating perturbation as the original T42L15 run (Fig. 1), with an increased westerly wind speed on the poleward side of the jet center and a decrease on the equatorward side. However, the magnitude of the response is around $75 \%$ larger than in the original experiment. This is consistent with Gerber et al.'s (2008) result that at T42 horizontal resolution, an increased vertical resolution results in a longer decorrelation time scale and thus an expected increase in the model sensitivity to external forcing. However, this trend is reversed when the resolution is increased to L60. This appears contrary to 


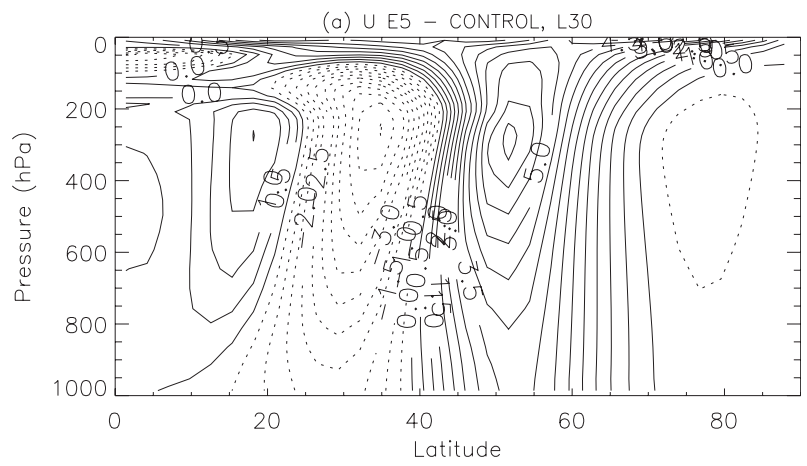

(b) U, E5 - CONTROL, TOPOGRAPHY

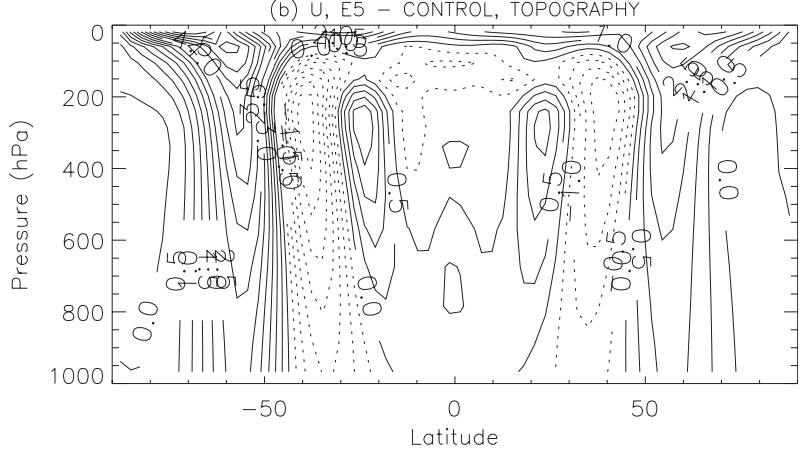

FIG. A1. Equilibrium difference in zonal mean zonal wind between the E5 heating case and the control run for (a) the T42L30 model and (b) a run with topography in the Northern Hemisphere.

Gerber et al. (2008), who found that the decorrelation time scale was insensitive to vertical resolution beyond L40 at T42 horizontal resolution. This suggests the need for more detailed investigation including the sensitivity to numerical aspects of different models.

For the run with topography, a ridge $2000 \mathrm{~m}$ in height oriented north-south has been placed in the Northern Hemisphere centered on $0^{\circ}$ longitude and $45^{\circ}$ latitude (i.e., placed across the latitude of the jet). The topography is elliptical in shape, with an eccentricity of 4 and a half-width of $20^{\circ}$ longitude. Figure A1b shows the zonal wind response to the E5 heating perturbation. Both hemispheres are shown because the model is no longer symmetric around the equator. A similar magnitude of response to the original E5 experiment is seen in the $\mathrm{SH}$ as expected. In the hemisphere with the topography the magnitude of the response is reduced (by around $50 \%$ ) but the pattern of response is, again, qualitatively the same.

These experiments show that the magnitude of the model response is sensitive to aspects of the model specification. Nevertheless, preliminary analysis of other meteorological fields, notably the eddy momentum flux, indicates that the same mechanisms operate. The reasons for the differing magnitudes of the response are an area for future study.

\section{REFERENCES}

Baldwin, M. P., and T. J. Dunkerton, 2005: The solar cycle and stratosphere-troposphere dynamical coupling. J. Atmos. Sol.Terr. Phys., 67, 71-82.

Chen, G., and I. M. Held, 2007: Phase speed spectra and the recent poleward shift of Southern Hemisphere surface westerlies. Geophys. Res. Lett., 34, L21805, doi:10.1029/2007GL031200.

,-- , and W. A. Robinson, 2007: Sensitivity of the latitude of the surface westerlies to surface friction. J. Atmos. Sci., 64, 2899-2915.

Chen, P., and W. A. Robinson, 1992: Propagation of planetary waves between the troposphere and stratosphere. J. Atmos. Sci., 49, 2533-2545.

Crooks, S. A., and L. J. Gray, 2005: Characterization of the 11-year solar signal using a multiple regression analysis of the ERA-40 dataset. J. Climate, 18, 996-1015.

Edmon, H. J., Jr., B. J. Hoskins, and M. E. McIntyre, 1980: Eliassen-Palm cross sections for the troposphere. J. Atmos. Sci., 37, 2600-2616.

Gerber, E. P., and G. K. Vallis, 2007: Eddy-zonal flow interactions and the persistence of the zonal index. J. Atmos. Sci., 64, 32963311.

—, S. Voronin, and L. M. Polvani, 2008: Testing the annular mode autocorrelation time scale in simple atmospheric general circulation models. Mon. Wea. Rev., 136, 1523-1536.

Gleisner, H., and P. Thejll, 2003: Patterns of tropospheric response to solar variability. Geophys. Res. Lett., 30, 1711, doi:10.1029/ 2003 GL017129.

Haigh, J. D., 1996: The impact of solar variability on climate. Science, 272, 981-984.

_ 1999: A GCM study of climate change in response to the 11year solar cycle. Quart. J. Roy. Meteor. Soc., 125, 871-892.

, 2003: The effects of solar variability on the Earth's climate. Philos. Trans. Roy. Soc. London, A361, 95-111.

_ - M. Blackburn, and R. Day, 2005: The response of tropospheric circulation to perturbations in lower-stratospheric temperature. J. Climate, 18, 3672-3685.

Hartmann, D. L., and P. Zuercher, 1998: Response of baroclinic life cycles to barotropic shear. J. Atmos. Sci., 55, 297-313.

Haynes, P. H., 2005: Stratospheric dynamics. Annu. Rev. Fluid Mech., 37, 263-293.

- M. E. McIntyre, T. G. Shepherd, C. J. Marks, and K. P. Shine, 1991: On the "downward control" of extratropical diabatic circulations by eddy-induced mean zonal forces. $J$. Atmos. Sci., 48, 651-678.

Held, I. M., and M. J. Suarez, 1994: A proposal for the intercomparison of the dynamical cores of atmospheric general circulation models. Bull. Amer. Meteor. Soc., 75, 1825-1830.

Hoskins, B. J., and A. J. Simmons, 1975: A multilayer spectral model and the semi-implicit method. Quart. J. Roy. Meteor. Soc., 101, 637-655.

Karoly, D. J., and B. J. Hoskins, 1982: Three-dimensional propagation of planetary waves. J. Meteor. Soc. Japan, 60, 109-123.

Kristjánsson, J. E., A. Staple, J. Kristiansen, and E. Kaas, 2002: A new look at possible connections between solar activity, clouds and climate. Geophys. Res. Lett., 29, 2107, doi:10.1029/ 2002GL015646.

Kushner, P. J., and L. M. Polvani, 2004: Stratosphere-troposphere coupling in a relatively simple AGCM: The role of eddies. $J$. Climate, 17, 629-639.

— , and - 2006: Stratosphere-troposphere coupling in a relatively simple AGCM: Impact of the seasonal cycle. $J$. Climate, 19, 5721-5727. 
Labitzke, K., and Coauthors, 2002: The global signal of the 11-year solar cycle in the stratosphere: Observations and models. $J$. Atmos. Sol.-Terr. Phys., 64, 203-210.

Larkin, A., J. D. Haigh, and S. Djavidnia, 2000: The effect of solar UV irradiance variations on the earth's atmosphere. Space Sci. Rev., 94, 199-214.

Leith, C. E., 1975: Climate response and fluctuation dissipation. $J$. Atmos. Sci., 32, 2022-2026.

Lorenz, D. J., and D. L. Hartmann, 2003: Eddy-zonal flow feedback in the Northern Hemisphere winter. J. Climate, 16, 12121227.

_ , and E. T. DeWeaver, 2007: Tropopause height and zonal wind response to global warming in the IPCC scenario integrations. $J$. Geophys. Res., 112, D10119, doi:10.1029/2006JD008087.

Lu, H., M. J. Jarvis, H.-F. Graf, P. C. Young, and R. B. Horne, 2007: Atmospheric temperature responses to solar irradiance and geomagnetic activity. J. Geophys. Res., 112, D11109, doi:10.1029/2006JD007864.

Matsuno, T., 1970: Vertical propagation of stationary planetary waves in winter Northern Hemisphere. J. Atmos. Sci., 27, 871-883.

Matthes, K., Y. Kuroda, K. Kodera, and U. Langematz, 2006: Transfer of the solar signal from the stratosphere to the tro- posphere: Northern winter. J. Geophys. Res., 111, D06108, doi:10.1029/2005JD006283.

Polvani, L. M., and P. J. Kushner, 2002: Tropospheric response to stratospheric perturbations in a relatively simple general circulation model. Geophys. Res. Lett., 29, 1114, doi:10.1029/ 2001 GL014284.

Salby, M. L., and P. F. Callaghan, 2006: Evidence of the solar cycle in the tropical troposphere. J. Geophys. Res., 111, D21113, doi:10.1029/2006JD007133.

Shepherd, T. G., 2002: Issues in stratosphere-troposphere coupling. J. Meteor. Soc. Japan, 80, 769-792.

Simmons, A. J., and D. M. Burridge, 1981: An energy and angularmomentum conserving vertical finite-difference scheme and hybrid vertical coordinates. Mon. Wea. Rev., 109, 758-766.

Son, S.-W., and Coauthors, 2008: The impact of stratospheric ozone recovery on the Southern Hemisphere westerly jet. Science, 320, 1486-1489.

Song, Y., and W. A. Robinson, 2004: Dynamical mechanisms for stratospheric influences on the troposphere. J. Atmos. Sci., 61, $1711-1725$

Williams, G. P., 2006: Circulation sensitivity to tropopause height. J. Atmos. Sci., 63, 1954-1961. 